\title{
REVISIÓN CRÍTICA DEL GÉNERO ARACHNOTHRYX PLANCH.
}

\author{
Attila L. Borhidi \\ Instituto de Biología, Universidad de Pécs, H-7624 Pécs, Ifjúság útja 6, Hungría \\ E-mail: borhidi@gamma.ttk.pte.hu
}

(Received 13 June, 2017; Accepted 31 July, 2017)

The last synthetic treatment of the Arachnothryx genus was published by D. Lorence in the Flora Mesoamericana (Vol. 4, part 2, 2012) based on a polyphyletic concept including several genera of different tribes and leaving the results of the molecular studies out of consideration. The analytic key offered by the author is based firstly on the highly complex and varied indumentum, and not on the more stable structural elements of the species, therefore the determination of the different species and species-groups is problematic. The present treatment is based on the original strictly monophyletic concept of Planchon (1849) pointed out and detailed by Steyermark, including into the study all (103) the valid species existing in México, Mesoamerica and South America. The new analytic key comprises all the existing Mexican and Mesoamerican species (81).

Key words: Arachnothryx, Flora Mesoamericana, Guettardeae, Rubiaceae

\section{HISTORIA BREVE DE LOS ESTUDIOS DEL GÉNERO ARACHNOTHRYX PLANCH.}

Como fue expuesto por Borhidi y Fernández (1981: 309) el concepto del género Rondeletia L. ha sido notablemente extendido por De Candolle (Prodr. 4: 406. 1830) y Bentham (Pl. Hartw. 1841). Planchon fue el primero, quien reconoció diferencias importantes en las flores de distintas especies de Rondeletia existentes en Centro América, que permiten su separación en géneros nuevos: Rogiera y Arachnothryx.

El nombre genérico Arachnothryx aparece por la primera vez en el tomo 5, página no. 442 de Flores des Serres en 1849. La página está dedicada a la 
descripción detallada del género Rogiera Planchon con 5 especies, basado en Rogiera amoena como especie típica, presentada con una ilustración de color también y separado de las Rondeletias verdaderas, listando Rondeletia odorata Jacq., $R$. umbellata Sw., $R$. tomentosa Sw. (= R. stipularis (L.) Druce), $R$. laevigata Ait., $R$. parviflora Poir. y R. brevipes Benth. (= R. pubescens HBK). El nombre Arachnothryx está mencionado solamente en la primera Observación en paréntesis, como nombre genérico para separar un grupo de especies distintas de las Rondeletias enumeradas arriba, caracterizado por tener corola 4-mera, con tubo aracnoideo-tomentoso y garganta abierta con anillo faucial no observable y representado por Rondeletia buddleioides Benth. (Arachnothryx buddleioides), Rondeletia laniflora Benth. (Arachnothryx laniflora), Rondeletia reflexa Benth. (Ar. reflexa), Rondeletia discolor HBK. (Ar. discolor?), Rondeletia leucophylla HBK. (Ar. leucophylla), y Rondeletia elongata Bartl. ex DC. (Ar. elongata? actualmente: Arachnothryx leucophylla).

La separación de los géneros propuestos por Planchon no fueron aceptados por los autores posteriores (Bentham et Hooker 1873, Hemsley 1879, Schumann 1897, Standley 1918) durante más de un siglo. Standley creando la versión clásica del tratamiento del género Rondeletia en el primer tomo de las Rubiáceas en la Flora de Norte América (1918) quisó mantener su unidad en sentido amplio, pero tenía que confrontarse con su riqueza morfológica enorme también. La solución fue, que dividió el género en 15 grupos, incluyendo los géneros Rogiera como sección Amoenae, y Arachnothryx como sección Leucophyllae y sección Laniflorae.

La resurrección del género Arachnothryx fue efectuada por Steyermark (1967) quien trabajando sobre las Rubiáceas de la Guayana Venezolana encontró, que la corola 4-mera, tomentosula con garganta abierta y fruto septicidal corresponde completamente a la descripción del género Arachnothryx en no menos de 21 especies y estos caracteres lo separan claramente de las especies de Rondeletia existentes en la misma area, pero teniendo flores 5-meras, tubo glabro y garganta estrechada por un anillo lamelar discolor y cápsula loculicida. En una nota Steyermark menciona también, que la Rondeletia amoena pertenece a un otro género también.

En la edición de la Flora de Guatemala (1975) resultó una situación desgraciada por el rechazo de los argumentos de Steyermark. Standley, el primer autor ya no vivió (murió en 1963, antes de la publicación de Steyermark), L. O. Williams, el segundo autor no se consideró aurorizado para hacer mayores cambios en el manuscrito y publicó la declaración siguiente: “Dr. Julián A. Steyermark in his study of the Rubiaceae in Mem. N.Y. Bot. Gard. 17: 241-261. 1967 has separated Arachnothryx Planchon out of Rondeletia and has given reasons, in a key, for doing this. Several of the species in this flora and, 
in fact, perhaps most of them would need to be transferred to Arachnothryx if the separation were followed."

La verdad es, que la Flora de Guatemala taxonómicamente no fue preparado para utilizar los criterios de Steyermark, porque no dieron importancia a la dehiscencia de frutos: entre las 32 especies de "Rondeletia" tratadas solamente una (Rondeletia amoena = Rogiera amoena) tiene indicada la dehiscencia del fruto. Con la declaración desdichosa los autores crearon un ejemplo peligroso del voluntarismo. Voluntarismo en mi glosario es el caso, cuando un experto no deja influirse por las evidencias científicas negligándolas y toma decisiones con intención no impecables o anacronísticas desde del punto de vista científico. Es la situación cuando la opinión personal sobreescribe la objetividad.

Lorence (1991) describe que "In most modern floristic treatment (e.g., Dwyer 1980; Kirkbride 1969; Standley y Williams 1975) Arachnothryx and Rogiera have been considered as synonyms of Rondeletia". Lorence confundió la publicación reciente con la publicación moderna. Estar moderno es cuestion del concepto y no de la fecha de la publicación. En la realidad, los tratamientos citados por Lorence son repeticiones del concepto de Standley, publicado en 1918.

El tratamiento verdaderamente moderno era la sugerencia de Steyermark seguido por Borhidi (1982) y con M. Fernández, cómo estos han sido confirmados posteriormente por los análises molecular-filogenéticos. Ellos circunscribieron el género Rondeletia basado en la especie típica del género: Rondeletia americana L. (1982a), una especie aislada en Jamaica y Las Antillas Menores. En el marco de un estudio amplio encontraron, que los caracteres florales y frutales más importantes de la especie típica se encuentran en más de 100 especies distribuidas en las Antillas Mayores, mientras que en América Central hay solamente 3 especies del género Rondeletia, la Rondeletia belizensis en Mexico, Belize y Guatemala, y la Rondeletia hameliifolia y la Rondeletia panamensis en Panamá.

El duelo sobre la validez del género Arachnothryx empezó en el papel de David Lorence en el volumen inaugural de la revista Novon en 1991. El no tenía corazón para atacar a Steyermark por la revalidación del género Arachnothryx, sino atacó a Borhidi, que parecía un adversario más fácil (y europeo, no americano) escribiendo los siguientes: "Borhidi and his collaborators proposed a very narrow generic concept for Rondeletia limiting its scope to include about a hundred West Indian species." "While studying Mexican and Central American Rondeletia I encountered species with virtually every possible combinations of morphological characters used to separate Arachnothryx, Rogiera and Rondeletia s. str. Beause of the existence of intermediate taxa... I believe at present it is unfeasible to recognize Arachnothryx, Rogiera y Javorkaea 
as distinct from Rondeletia". Los ejemplos de los intermediate taxa enumerados por Lorence para ilustrar las transiciones resultaron incorrectos porque representan distintos géneros pertenecientes a diversas tribus o porque estan basados en observaciones superficiales. Lorence en este artículo se presentó como un confusionista, que buscando las transiciones en toda parte confunde la variación de los caracteres individulales de poco valor taxonómico con la combinación de caracteres estable, que tiene un valor decisivo en las separaciones taxonómicas. Confunde también la dehiscencia loculicida y septicida inventado la dehiscencia intermediaria, cuando "dehiscence is initially loculicidal than septicidal" que es nada más que un proceso especial de la dehiscencia septicidal. Después de esta discusión Lorence describió 11 especies y 2 combinaciones nuevas de Rondeletia, que actualmente están reconocidas como 12 especies de Arachnothryx y 1 de Rogiera. Dos años más tarde en 1993 describió 3 Rondeletias nuevas más en la Flora Costaricensis de Burger y Taylor, que todas pertenecen al género Arachnothryx.

El mismo concepto prevaleció el Nomenclator of Mexican and Central American Rubiaceae (1999), aunque el autor tenía ocho años de preparación para estudiar los caracteres morfológicos del género Rondeletia. Es imprecendible estudiar el tipo de un género antes de ubicar docenas de especies nuevas en el, si uno quiere evitar el fracaso. Es extraño, que el autor pone 90 nombres de especies en la sinonimía sin explicación en la introducción y/o en las referencias citadas. Para corregir las decisiones taxonómicas discutibles Borhidi publicó una series de reflexiones críticas (2001).

Los estudios de las secuencias moleculares de las Rubiáceas saliendo desde el mismo año produjeron una serie de conocimientos nuevos de gran importancia poniendolos en enfoque nuevo (p. e. . En el caso del género Rondeletia el concepto amplio sufrió un gran cambio (Rova 1999, Rova et al. 1999, 2002), la existencia de los Arachnothryx y Rogiera como géneros válidos ha sido reconfirmada y su distinción del género Rondeletia claramente determinada. En 2003 el Index Kewensis también legalizó la validez de los tres géneros cómo distintos.

En 2004 recibí una carta electrónica desde Lorence con el texto siguiente: "You will undoubtedly pleased to hear that I have decided to adopt the Rondeletieae segregate genera, Arachnothryx and Rogiera as well as Rondeletia s. str. for the Flora Mesoamericana treatment". Finalmente Lorence tenía que aceptar el "concepto genérico muy estrecho de Borhidi y collaboradores", y por esto ellos tienen que sentirse feliz. No es muy elegante menospreciar el concepto de un colega en una publicación internacional y despues reconocer en una carta privada que el concpto del colega es correcto y hay que aceptarlo en la Flora Mesoamericana. 
En 2004 Borhidi y colaboradores publicaron un artículo crítico e sintético del todo el complejo de Rondeletia s. 1. separando del Arachnothryx el género nuevo Renistipula con 3 especies mexicanas y mesoamericanas. En la base de los estudios moleculares (Rova 1999, Rova et al. 1999, 2002) reconocieron diferencias morfológias importantes entre las especies del género Rogiera que permitieron la separación del género nuevo Rovaeanthus con 2 especies de México y Mesoamérica.

Es interesante, que durante de veinte años de discusión sobre la taxonomía del género Rondeletia s. 1. varios autores (Borhidi y Fernández 1982, Borhidi y Darók 2001, Borhidi et al. 2004, 2011, Bremer 2009, Bremer et al. 1995, Darók et al. 2001, Kocsis et al. 2004, Manns y Bremer 2010, Rova 1999, Rova et al. 1999, 2002, 2009, Stranczinger et al. 2006, 2010) realizaron una serie de investigaciones morfológicas, anatómicas, submicroscópicas y moleculares enriqueciendo los conocimientos con numerosas pruebas nuevas utilizables para la distinción de las unidades diferentes taxonómicas, mientras Lorence se portaba como un juez, aceptando o rechazando y negligando los resultados y conceptos nuevos, sin hacer un estudio própio original.

El último tratamiento sintético del género apareció en la Flora Mesoamericana, tomo 4. parte 2. compilado por Lorence, quién en esta obra siguió su actividad confusionística, cambiando la Rondeletia polifiética en Arachnothryx polifilético incluyendo 4 distintos géneros pertenecientes en 3 diversas tribús (Arachnothryx y Javorkaea: Guettardeae; Donnellyanthus: Rondeletieae; Renistipula: Hamelieae). Amplió incorrectamente el protólogo original de Planchon y Steyermark con caracteres ajenos, no existentes en las especies pertenecientes al género formando un "cajón de sastre" (termino prestado de Lorence), pués el Arachnothryx Planch. sensu Lorence no es idéntico con Arachnothryx Planch. y debe cualificarse cómo um homónimo posterior ilegítimo. Otro nombre ilegítimo creado por Lorence es Arachnothryx armentalis (L. O. Wms.) Lorence que es un binómio posterior del Arachnothryx ovandensis (Lundell) Borhidi. El tratamiento es incompleto y erróneo porqué incluye 7 especies no existentes en Mesoamérica, (solamente esperadas por el autor), mientras faltan 4 especies descritas en 2005 y 2006 de Tabasco y Chiapas por Borhidi y colaboradores y pusó en sinonimía incorrectamente 4 especies reconocidas cómo válidas (véase abajo). La clave ofrecida por Lorence para determinar las especies está basada mayormente en la pubescencia muy variada y compleja de distintos órganos, dando menos importancia a los elementos estructurales más estables de las plantas. Por esto la utilización de la clave oculta muchas dificultades e incertidumbres.

Para tener una visión más estable y taxonómicamente más objetiva sobre este género notable de la familia Rubiaceae regresamos en este tratamiento al concepto monofilético original de Planchon (1849) reactivado y circunscrito 
correctamente por Steyermark, y aplicándolo a las 81especies existentes en la flora de México y Mesoamérica.

\section{Arachnothryx Planch. 1849}

Arachnothryx Planch., Fl. Serres 5: tab. 442. (1849). Tipo: Arachnothryx leucophylla (Kunth) Planch. Syn.: Rondeletia sensu Standley, N. Amer. Fl. (1918). et auct. Amer. p.p. non L.

Arbustos o arbolitos a veces sufrútices hermafroditas, inermes. Estípulas interpeciolares triangulares, 1-aristadas, rara vez brevemente bidentadas, frecuentemente con coléteres marginales o/y interiores, mayormente persistentes. Hojas opuestas, raras veces ternadas o verticiladas, isófilas o anisófilas, con o sin domácios, nervadura menor reticulada, excepcionalmente lineolada. Inflorescencias terminales o raras veces axilares, cimosas, espiciformes, tirsoideas, corimbosas a veces paniculadas con un eje primario bien desarrollado, frecuentemente monocasiales, dicasiales o escorpioideas, mayormente multifloras y bracteadas. Flores 4-meras homostilas o distilas; limbo calicino 4-lobulado, lóbulos generalmente desiguales, 1 o 2 a veces foliáceos. Corola hipocrateriforma, rara vez embudada, blanca, amarilla, anaranjada, roja a purpurea; la garganta abierta, glabra, sin engrosamiento de color distinto o anillo de pelos amarillos, (excepcionalmente blanco-puberulenta, nunca estrigiloso barbada) tubo corolino peloso por dentro, en o cerca de la base, a menudo aracnoide-tomentoso o glabrescente por fuera, lóbulos siempre 4 , imbricados. Estambres 4, anteras dorsifijas inclusas en la garganta, sésiles o subsésiles. Estilo glabro. Estigmas 2 o 1 a 2-lobados, lóbulos lineares. Disco del ovario glabro, excepcionalmente pulverulento, nunca estrigiloso-hirsuto, más corto que el tubo del cáliz. Fruto en cápsula 2-locular, aovado u oblongo, con dehiscencia septicida, (a veces loculicida en la fase inicial de la eprtura); placenta elíptica o aovada con una inserción central. Semillas numerosas triangulares o romboides, comprimidas, no aladas, con células penta- a hexagonales \pm isodiamétricas y paredes perpendiculares engrosadas y duplicadas sin ornamentación. Número básico de cromosomas $\mathrm{x}=9$.

Género neotropical con unas 100 especies en México, América Central y Norte de Sudamérica hasta Brasil y Perú con centro de diversificación en México y Guatemala.

Según los estudios moleculares (Rova et al. 2009, Manns y Bremer 2010) el género Arachonthryx pertenece a la tribu Guettardeae.

1a Inflorescencia panícula estrecha de forma de espiga alargada compuesta por ramas cimosas o racemosas, a menudo secundifloras

1b Inflorescencias de formas varias, no formas de espigas 
2a Corola aracnoide-tomentosa por fuera, a veces glabrescente 3

$2 \mathrm{~b}$ Corola de pubescencia diferente o glabra, no tomentosa por fuera $\quad 27$

3a Hojas sésiles, cordadas en la base 4

3b Hojas pecioladas, ramas de espigas no capitadas 5

4a Inflorescencia compuesta de inflorescencias parciales de capítulos densos, hojas blanco-hirsutas en el envés

A. mexicana

4b Inflorescencia compuesta de cimas secundifloras, hojas arachnoidetomentosas en el envés

A. sessilis

5a Hojas tomentosas en el envés, cuando jóvenes, glabrescentes con la edad, o con otro tipo de pelosidad

5b Hojas persistente y densamente tomentosas en el envés 17

$\begin{array}{lll}6 \text { 6a Tubo de la corola glabro } & 7\end{array}$

6b Tubo de la corola tomentoso 9

7a Lóbulos del cáliz iguales, de $2 \mathrm{~mm}$ de largo, lineares, agudos, iguales o más largos que el hipanto A. filisepala

$7 \mathrm{~b}$ Lóbulos del cáliz desiguales, hasta $1 \mathrm{~mm}$ de largo, ovadas, obtusas, más cortos que el hipanto 8

8a Estípulas persistentes, estrechamente triangulares, acuminadas y agudas; tubo de la corola 5 a $6 \mathrm{~mm}$ de largo, glabro por dentro A. gracilispica

$8 \mathrm{~b}$ Estípulas caedizas, aovadas, redondeadas y mucronuladas en el ápice; tubo de la corola 8 a $9 \mathrm{~mm}$ de largo, peloso en la base por dentro A. sooana

9a Lóbulos del cáliz desiguales con 1 expandido y 2 veces más largos, que los demás 3; hojas subsésiles con pecíolos de 1 a $4 \mathrm{~mm}$ de largo, obtusas, redondeadas a subcordadas en la base

A. secunda

9b Lóbulos del cáliz iguales o subiguales, ninguno expandido, hojas con pecíolos de 3 a $40 \mathrm{~mm}$ de largo, mayormente agudas o cuneadas en la base

10a Lóbulos del cáliz reflejos, igual o más largos que el hipanto 11

10b Lóbulos del cáliz más cortos que el hipanto 14

11a Tubo de la corola 6 a $10 \mathrm{~mm}$ de largo $\quad 12$

11b Tubo de la corola 12 a $19 \mathrm{~mm}$ de largo $\quad 13$ 
12a Inflorescencias pedunculadas delgadas, lóbulos del cáliz desiguales, tubo de la corola de 6 a $8 \mathrm{~mm}$

A. septicidalis

12b Inflorescencia sésil, ancho y corto, lóbulos del cáliz iguales tubo de la corola 9 a $10 \mathrm{~mm}$

A. hirtinerois

13a Hojas esparcidamente araneoso-tomentulosas en el envés; tubo de la corola de 12 a $15 \mathrm{~mm}$ de largo, lóbulos 2 a $3 \mathrm{~mm}$ de largo

A. skutchii

13b Hojas glabras en ambas caras; tubo de la corola de 16 a $19 \mathrm{~mm}$ de largo, lóbulos de 3 a $5 \mathrm{~mm}$

A. sousae

14a Corola blanca, el tubo de 5 a $7 \mathrm{~mm}$ de largo

A. myriantha

14b Corola roja a rojo color vino tinto, el tubo 7 a $9 \mathrm{~mm}$ de largo

15a Hojas lustrosas en el haz, blanco tomentosas en los nervios del envés, nervios laterales 5 a 8 pares

A. wendtii

15b Hojas no lustrosas en el haz, esparcida-tomentulosas o glabras en el envés, nervios laterales 8 a 10 pares

16a Estípulas 1.5 a $6 \mathrm{~mm}$ de largo, aovado-triangulares, subuladas, hojas glabras en ambas caras, inflorescencias de 1 a $2 \mathrm{~cm}$ de ancho; cápsulas 1.5 a $2.5 \mathrm{~mm}$ de diámetro

A. gracilis

16b Estípulas 7 a $9 \mathrm{~mm}$ de largo, lanceoladas, hojas esparcido-tomentulosas en el envés, inflorescencias de 2 a $4 \mathrm{~cm}$ de ancho; cápsula 3 a $3.5 \mathrm{~mm}$ de diámetro

A. rubens

17a Hojas densamente velloso-pelosas en los nervios del envés $\quad 18$

$17 \mathrm{~b}$ Hojas tomentosas sin pelos hirsutos en el envés

18a Estípulas glabras por fuera, vellosas por dentro

A. manantlanensis

18b Estípulas vellosas o estrigosas por fuera

19a Estípulas de 10 a $20 \mathrm{~mm}$ de largo, vellosas en el haz, glabras en el envés, corola blanca

A. villosa

19b Estípulas de 7 a $11 \mathrm{~mm}$ de largo, estrigosos en ambas caras, corola rosada, rojo-violeta a roja, fragante

A. nebulosa

20a Pedúnculo y raquis aracnoide-blanco-tomentosos

20b Pedúnculo y raquis de la inflorescencia ferrugíneo-hirsutos o tomentosos 
21a Ramas inferiores de las inflorescencias notablemente más largas que las superiores

21b Ramas inferiores de las inflorescencias \pm iguales de largo que las superiores

22a Corola embudada, tubo 3 a $4 \mathrm{~mm}$ de largo, abruptamente ensanchado, lóbulos desiguales de 2 a $2.5 \mathrm{~mm}$ de largo

A. breviflora

22b Corola hipocrateriforma, tubo 10 a $12 \mathrm{~mm}$ de largo, no ensanchado notablemente, lóbulos de 2 a $3 \mathrm{~mm}$ de largo

A. pyramidalis

23a Tubo de la corola hasta $1 \mathrm{~cm}$ de largo

A. buddleoides

23b Tubo de la corola más largo

24a Hojas nítidas en el haz, nervios terciarios y cuaternarios obsoletos en el haz apenas visibles en el envés, flores corto-pediceladas, no secundas, bractéolas mínimas o ausentes, lóbulos del cáliz subiguales, triangulares, hasta $1 \mathrm{~mm}$ de largo

A. laniflora

24b Hojas mates y escabérulas en el haz; venación densa y visible en el haz, prominente en el envés; flores sentadas en cimas bifurcadas, secundas, cada flor subtendida con una bractéola linear persistente, hasta $5 \mathrm{~mm}$ de largo; lóbulos del cáliz desiguales, lineares, 1.5 a $4 \mathrm{~mm}$ de largo

A. bourgaei

25a Inflorescencias ferrugíneo-tomentosas

A. tabascensis

25b Pedúnculo y raquis ferrugíneo-hirsutos 26

26a Lóbulos del cáliz iguales, ovados, $0.5 \mathrm{~mm}$ de largo

A. ovandensis

26b Lóbulos del cáliz desiguales, lineares, 1 a $3 \mathrm{~mm}$ de largo

A. monticola

27a Hojas densamente estrigulosas o hirsutas en la nervadura del envés 28

27b Hojas glabras o seríceas a adpreso-pelosas en el envés

28a Hojas subsésiles, cordadas en la base, espiga compuesta de inflorescencias parciales de capítulos densos

A. ginetteae

28b Hojas pecioladas, flores no en capítulos densos

29a Hojas largamente atenuadas y decurrentes hasta la base del pecíolo; inflorescencias más cortas que las hojas que subtienden

A. thiemei

29b Base de las hojas no decurrentes hasta la base del pecíolo; inflorescencias inflorescencias más largas que las hojas que subtienden 
30a Hojas estrigoso-hirsutas en el haz, inflorescencias deflexas a péndulas lóbulos de cáliz subulados, agudos

A. tacanensis

30b Hojas glabras y nitidas en el haz (excepto de pelos esparcidos en el nervio medio); inflorescencias erectas, lóbulos del cáliz obtusos a redondeados 31

31a Tallo, envés de las hojas e inflorescencia ferrugíneo-hirsutos, hojas lanceoladas lóbulos del cáliz ovados

A. rufescens

31b Tallo, envés de las hojas e inflorescencia seríceo-estrigosos a vellosos, hojas elípticas de más de $10 \mathrm{~cm}$ de largo, lóbulos del cáliz obovados a orbiculares

A. tenorioi

32a Planta completamente glabra, hipanto glabro

A. gonzaleoides

32b Hipanto estriguloso, hirsuto o velloso

33a Tubo de corola glabro por fuera, setoso-pelosa en la base de los lóbulos; hojas adpreso-seríceas en los nervios del envés

33b Tubo de la corola estrigoso a peloso por fuera, hojas glabras o estrigosas 35

34a Estípulas unidas en la base en una vaína de 1 a $3 \mathrm{~mm}$ de largo, lóbulos del cáliz subiguales, de 0.5 a $1 \mathrm{~mm}$ de largo, más cortos que el hipanto

A. brenesii

34b Estípulas libres en la base, lóbulos del cáliz desiguales, los 3 menores de 0.7 a $3 \mathrm{~mm}$, el mayor 1,2 a $4 \mathrm{~mm}$ de largo, más largos que el hipanto

A. stachyoidea

35a Panículas mayormente axilares y más cortas que las hojas

35b Panículas todas terminales, a menudo más cortas que las hojas que subtienden

36a Lóbulos del cáliz triangulares, de $0.5 \mathrm{~mm}$ de largo más cortos que el hipanto; tubo del cáliz de 3 a $4 \mathrm{~mm}$ de largo

A. heteranthera

36b Lóbulos del cáliz elípticas a lineares de 2 a $6 \mathrm{~mm}$ de largo, más largos que el hipanto. Tubo de la corola de 8 a $11 \mathrm{~mm}$ de largo

A. sanchezii

37a Inflorescencia muy estrecha y densa, cimas laterales sésiles o subsésiles 38

$37 \mathrm{~b}$ Inflorescencia más laxa, cimas laterales pedunculadas

38a Hojas iguales en un par por tamaño, domaciadas en el envés, cimas laterales sésiles, bractéolas pequeñas o incospícuas, lóbulos del cáliz de $1 \mathrm{a}$ $1.5 \mathrm{~mm}$ de largo, subiguales

A. secundiflora 
38b Hojas mayormente desiguales en un par por tamaño, sin domácios, cimas con bractéolas de 4 a $6 \mathrm{~mm}$ de largo, lóbulos del cáliz desiguales

39a Estípulas triangulares de 2 a $3 \mathrm{~mm}$ de largo, glabras por fuera; lóbulos menores del cáliz de 2 a $3 \mathrm{~mm}$ el mayor 3.5 a $5.5 \mathrm{~mm}$ de largo, corola roja o púrpura

A. angustispica

39b Estípulas triangular-subuladas de 4 a $6 \mathrm{~mm}$ de largo, pubérulas por fuera; lóbulos menores del cáliz de hasta $1 \mathrm{~mm}$, el mayor 2 a $3 \mathrm{~mm}$ de largo, corola blanca o rosada

A. pansamalana

40a Eje de la inflorescencia cilíndrico, brácteas mucho más cortos que las cimas laterales, lóbulos del cáliz linear-lanceolados

A. capitellata

a) Ramas, hojas e inflorescencia densamente hirsutas, lóbulos del cáliz desiguales de 1 a $2.5 \mathrm{~mm}$ de largo, tubo de la corola de 9 a11 $\mathrm{mm}$ de largo, hirsuto

ssp. capitellata

b) Ramas, hojas e inflorescencias esparcidamente estrigosas, lóbulos del cáliz subiguales de 0.2 a $1.2 \mathrm{~mm}$ de largo, tubo de la corola 4 a $8 \mathrm{~mm}$ de largo, adpreso estriguloso

ssp. pringlei

40b Eje de la inflorescencia anguloso, apretado ferrugíneo-peloso, brácteas más largas que las cimas laterales, lóbulos del cáliz desiguales, 1 mayor oblongo-elíptico a espatulado

A. linguiloba

41a Hojas a menudo ternadas

41b Hojas siempre opuestas

42a Hojas estrigulosas o araneoso-tomentosas en el envés, corola estrigulosa o estriguloso-serícea a pubérula por fuera

42b Hojas con acumen muy estrechas, largas y falcadas, glabras en el envés, pelosas en el nervio medio, corola glabra por fuera A. monteverdensis

43a Hojas brevemente pecioladas, subiguales o poco desiguales, estriguloso hirsutas en los nervios del envés, tubo de la corola 13 a $14 \mathrm{~mm}$

A. chiriquiana

43b Hojas sésiles o subsésiles, muy desiguales, araneoso-tomentosas en el envés; tubo de la corola 10 a $11 \mathrm{~mm}$ de largo

A. povedae

44a Hojas araneoso-tomentosas en el envés

44b Hojas no araneoso-tomentosas en el envés

45a Hojas tomentosas en el envés cuando jóvenes, glabrescentes con la edad, o con otro tipo de pelosidad 
45b Hojas persistente y densamente tomentosas en el envés

46a Inflorescencias sentadas, flores en corimbos laxos

A. flocculosa

$46 \mathrm{~b}$ Inflorescencias pedunculadas

47a Inflorescencia paniculada, o tirsoideo-piramidal, multiflora $\quad 48$

$47 \mathrm{~b}$ Inflorescencia corimbosa, umbelada o capitada

48a Estípulas triangulares de 2 a $3 \mathrm{~mm}$ de largo; inflorescencias axilares, corola bicolor con tubo rojo y lóbulos rosados

A. michoacana

48b Estípulas triangular-subuladas de 4 a $11 \mathrm{~mm}$, con una vaina basal de 1 a $4 \mathrm{~mm}$ de largo; inflorescencias terminales, corola concolor, blanca o rosada

A. bertieroides

49a Flores en capítulos o corimbos densos, multifloros, hipanto y cáliz densamente blanco-vellosos o tomentosos

A. leucophylla

49b Flores en corimbos o umbelas delgadas y laxas, 5 a 9-floras, hipanto y cáliz glabrescentes a glabros

A. pauciflora

50a Inflorescencia compuesta por cimas bífidas, arregladas en un rácimo, flores sésiles secundas, estípulas bidentadas, hojas ásperas en el haz

A. rekoi

50b Inflorescencia capitada, subcapitada, cimoso- o tirsoideo -paniculada, flores mayormente pediceladas, no secundas, estípulas enteras, hojas no ásperas en el haz

51a Inflorescencias sésiles o subsésiles con pedúnculos de hasta $1 \mathrm{~cm} \quad 52$

$51 \mathrm{~b}$ Inflorescencias pedunculadas con pedúnculos de 1 a $4 \mathrm{~cm}$ de largo 53

52a Lóbulos del cáliz lanceolados, 8 a $13 \mathrm{~mm}$ de largo, tubo de corola 14 a 15 mm de largo

A. darienensis

$52 \mathrm{~b}$ Lóbulos del cáliz triangular-subulados u obovado elípticos de 1 a $5 \mathrm{~mm}$ de largo; tubo de la corola de 8 a $13 \mathrm{~mm}$ de largo

A. dwyeri

53a Lóbulos del cáliz orbicular-oblongos aovado- u obovado-deltados, 0.5 a $4 \mathrm{~mm}$ de largo

53b Lóbulos del cáliz lineares u oblanceolados, 3 a 6 mm de largo

54a Hojas flocculoso-tometosas y rugulosas en el haz, pardúzco-tomentosas en el envés, lóbulos del cáliz de 2 a $4 \mathrm{~mm}$ de largo

A. evansii

54b Hojas glabrescentes a glabras y lustrosas en el haz, blanco-tomentosas en el envés, lóbulos del cáliz 0.5 a $2 \mathrm{~mm}$ de largo

A. nitida 
55a Hipanto y tubo de la corola aracnoide-tomentosos

A. leucophylla

55b Hipanto y tubo de la corola largamente albo-vellosos

A. guerrerensis

56a Estípulas aovadas, oblongo-obovadas, o espatulado-oblongas, de 6 a 17 $\mathrm{mm}$ de largo, persistentes

56b Estípulas pequeñas, agudas, subuladas a mucronadas, a menudo caducas

57a Hojas coriáceas a subcoriáceas, glabras en ambas caras

A. linguiformis

57b Hojas cartáceas, hirsutas a vellosas en ambas caras 58

58a Tubo de la corola 30 a $32 \mathrm{~mm}$, lóbulos de 10 a $15 \mathrm{~mm}$ de largo

A. megalantha

58b Tubo de la corola 13 a $19 \mathrm{~mm}$ de largo

59a Ápice de las hojas rectas; eje de las inflorescencias e hipanto hirsutotomentosos, lóbulos de la corola ovados a elípticos de 4 a 9 mm de largo

A. uxpanapensis

59b Ápice de las hojas falcadas; eje de las inflorescencias e hipanto estrigososeríceos, lóbulos de la corola suborbiculares de 2.5 a $3 \mathrm{~mm}$ de largo

\section{A. chaconii}

60a Lóbulos de la corola 1.5 a $4 \mathrm{~mm}$ de largo, la garganta de la corola glabra 61

60b Lóbulos de la corola 5 a $9 \mathrm{~mm}$ de largo, la garganta de la corola y/o los senos de los lóbulos barbados o tomentulosos hasta pelosos con pelos blancos 80

61a Tubo de la corola glabra por fuera

$61 \mathrm{~b}$ Tubo de la corola pubescente por fuera

62a Hojas lineoladas con nervios transversales paralelos en el haz A. lineolata

$62 \mathrm{~b}$ Hojas no lineoladas

63a Lóbulos del cáliz subiguales, filiformes

63b Lóbulos del cáliz desiguales; hojas e hipanto glabros, 1 lóbulo del cáliz foliáceo, espatulado a elíptico

A. purpurea

64a Ramitas cilíndricas y glabras, hojas glabras con reticulación de venas visibles en ambas caras, inflorescencia, hipanto y cáliz glabros

A. jurgensenii

64b Ramitas angulosas e hirsutas, hojas hirsutas, reticulación de venas no visible en el haz, inflorescencia, hipanto y cáliz hirsutos a vellsos

A. jaliscensis 
65a Inflorescencias parciales en forma de capítulo o corimbo estrecho 66

65b Inflorescencias parciales no en forma de capítulo o corimbo estrecho 72 66a Hojas oblongo-elípticas, obtusas o redondeadas en el ápice A. chinajensis

66b Hojas acuminadas y agudas en el ápice $\quad 67$

67a Lóbulos del cáliz subiguales $\quad 68$

67b Lóbulos del cáliz desiguales $\quad 69$

68a Hojas de 2 a 4 por 1.2 a $4 \mathrm{~cm}$, con nervios laterales 3 a 4 pares, inflorescencias con pedúnculos de 5 a $15 \mathrm{~mm}$, tubo de corola 12 a $14 \mathrm{~mm}$, glabro en la garganta

A. atravesadensis

68b Hojas más grandes, escabrosas en el haz, nervios laterales 5 a 7 pares, inflorescencias sésiles o subsésiles, corola tube 9 a $11 \mathrm{~mm}$, reloso alrededor de la garganta

A. scoti

69a Hojas sin domácios en el envés $\quad 70$

69b Hojas con domácios en el envés $\quad 71$

70a El lobo calicino mayor obovado-elíptico a elíptico de 4 a $6 \mathrm{~mm}$, 2 veces más largo que los menores de1.5 a $3 \mathrm{~mm}$, linear-subulados $A$. torresii

$70 \mathrm{~b}$ El lobo calicino mayor oblongo-linear, de 3 a $5 \mathrm{~mm}$, los menores estrechamente triangulares, de 1 a $3 \mathrm{~mm}$

A. calycosa

71a Ejes de la inflorescencia glabros, lobo mayor del cáliz oblongo-elíptico de 2 a $4.5 \mathrm{~mm}$, los menores linear-subilados de 1 a $2.5 \mathrm{~mm}$ de largo, cápsula subglobosa de 2 a $4.5 \mathrm{~mm}$ de diámetro, glabrada, no coronada por los lóbulos del cáliz

A. nelsonii

71b Ejes de la inflorescencia densamente fulvo-estrigulosos; lobo mayor del cáliz lanceolado a ligulado de 9 a $10 \mathrm{~mm}$, los menores linear-subulados de 2 a $5 \mathrm{~mm}$ de largo, cápsula elipsoidal, de 4 a $5 \mathrm{~mm}$ de diámetro, estrigilosa, coronada por los lóbulos del cálit persistentes A.chiapensis

72a Lóbulos del cáliz subiguales $\quad 73$

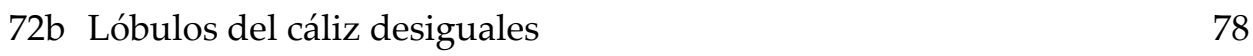

73a Lóbulos del cáliz lanceolados $\quad 74$

73b Lóbulos del cáliz ovados u obovados; tubo de la corola estrigosa o adpreso pubescente 
74a Inflorescencia 6 a $14 \mathrm{~cm}$ de largo; tubo de corola 11 a $12 \mathrm{~mm}$ de largo, vellosa con pelos largos

A. ricoi

74b Inflorescencia 1.5 a $2.5 \mathrm{~cm}$ de largo; tubo de corola 4 a $5 \mathrm{~mm}$ de largo, esparcidamente hirsuto

A. brachythyrsa

75a Hojas pubescentes en los nervios, lóbulos del cáliz obovados, 2.5 a $3 \mathrm{~mm}$ de largo

75b Hojas glabras en ambas caras, lóbulos del cáliz ovados, hasta $1 \mathrm{~mm}$ de largo

76a Hojas de 4 a $10 \mathrm{~cm}$ de largo, nervios laterales 5 a 6 pares domaciadas en el envés; ejes y pedúnculos de la inflorescencia blanco-hirsutos, corola 14 a $15 \mathrm{~mm}$ de largo

A. latiloba

76b Hojas de 6 a $20 \mathrm{~cm}$ de largo, nervios laterales 7 a 9 pares sin domácios, ejes y pedúnculos de la inflorescencia fulvo-velutinosos, corola 9 a 10 mm de largo

A. tayloriae

77a Inflorescencia axilar, con pedúnculos de 1.5 a $3 \mathrm{~cm}$ de largo, corola 15 a $17 \mathrm{~mm}$ de largo; disco velloso

A. sinaloae

77b Inflorescencia terminal, sésil, pedúnculo nulo; corola 10 a $12 \mathrm{~mm}$ de largo, disco glabro

A. subglabra

78a Tubo de la corola hirsuto-peloso, lóbulos del cáliz desiguales, 1 anchamente aovado

A. rzedowskii

78b Tubo de la corola e hipanto adpreso seríceo, lóbulos del cáliz desiguales, 1 más largo

79a Pedúnculos y ramas de las inflorescencias densamente estrigulosos, lóbulo mayor del cáliz ligulado, los demás lineares, estrigosos

A. tuxtlensis

79b Pedúnculos y ramas de las inflorescencias glabros, lóbulo mayor del cáliz anchamente espatulado, los demás ovados, glabrados A. chimalaparum

80a Planta glabra; corola discolor, de 15 a $17 \mathrm{~mm}$ de largo; el tubo y la garganta purpúreo, los lóbulos blancos, base de los lóbulos y la garganta aracnoid-tomentosa

A. jurgensenii

80b Plantas estriguloso-hirsutas o pubescentes

81a Lóbulos del cáliz subiguales, 8 a $15 \mathrm{~mm}$ de largo; tubo de la corola 18 a 25 mm de largo

A. macrocalyx

81b Lóbulos del cáliz desiguales, los mayores de 2 a $4 \mathrm{~mm}$ de largo; tubo de la corola 10 a $18 \mathrm{~mm}$ de largo

A. scabra 
1. Arachnothryx angustispica Borhidi, E. Martínez y Ramos, Acta Bot. Hung. 57: 17. (2015). - Tipo: México, Estado Veracruz, Mpio. Tezonapa, Ejido San Antonio, conglomerado 64447 del Inventario Nacional Forestal y de Suelo. Selva alta perennifolia con vegetación secundaria arbustiva. Coord.: $18^{\circ} 70.111^{\prime} \mathrm{N}, 96^{\circ} 87.556^{\prime} \mathrm{W}$, alt. $1130 \mathrm{msnm}$. Col.: J. Canek Ledesma Corral y Vladimir Campos, JCLC 965; holotipo: MEXU, isotipo: BP. - Ilustr.: Acta Bot. Hung. 57: 00. (2015). - Distr.: México: Veracruz, endémica.

2. Arachnothryx aspera (Standl.) Borhidi, Acta Bot. Acad. Sci. Hung. 28: 68. (1982). Bas.: Rondeletia aspera Standl., N.Amer.Fl. 32: 54. (1918). - Holotipo: Costa Rica. Col.: Pittier (3243) (US). - Ilustr.: Burger y Taylor, Fieldiana Bot. n.s. 33: 55. t. 41. (1993). - Distr.: Costa Rica, endémica.

3. Arachnothryx atravesadensis (Lorence) Borhidi, Acta Bot. Hung. 38: 139. 1993-94 (1995). - Bas.: Rondeletia atravesadensis Lorence, Novon 1: 137. Fig. 1A, B. (1991). - Tipo: México: Oaxaca, Cerro Atravesada-Cerro Azul, Arroyo Rana. Col.: T. MacDougall (s.n.), 6 Dec 1956; holotipo: MEXU 253128!. - Distr.: México: Oaxaca, endémica. Incluída erroneamente en la Flora Mesoamérica por D. Lorence.

4. Arachnothryx bertieroides (Standl.) Borhidi, Acta Bot. Acad. Sci. Hung. 28: 70. (1982). - Bas.: Rondeletia bertieroides Standl., Field Mus. Publ. Bot. 4: 267. (1929). - Holotipo: Panamá. Col.: Cooper (598) (F). - Ilustr.: Dwyer, Ann. Mo. Bot. Gard. 67: 473. t. 85. (1980). - Distr.: Costa Rica y Panama, endémica.

$=$ Rondeletia cooperi Standl., Publ. Field Mus. Hist Nat. Bot. Ser. 4: 267. (1929). झ Arachnothryx cooperi (Standl.) Borhidi, Acta Bot. Acad. Sci. Hung. 28: 69. (1982).

$=$ Rondeletia platysepala Standl., Ann. Mo. Bot. Gard. 27: 343. (1940). $\equiv$ Arachnothryx platysepala (Standl.) Borhidi, Acta Bot. Acad. Sci. Hung. 28: 70. (1982).

$=$ Rondeletia salicifolia subsp. brevicorolla J. H. Kirkbr., Ann. Mo. Bot. Gard. 55: 383. (1963). $\equiv$ Arachnothryx brevicorolla (J. H. Kirkbr.) Borhidi, Acta Bot. Acad. Sci. Hung. 28: 70. (1982).

= Rondeletia salicifolia Dwyer et M. V. Hayden, Phytologia 15: 58. (1967).

$=$ Rondeletia urophylla Standl. et L. O. Wms., Phytologia 26: 129. (1973). झ Arachnothryx urophylla (Standl. et L. O. Wms.) Borhidi, Acta Bot. Hung. 35: 311. (1989).

$=$ Rondeletia kirkbridei Dwyer, Ann. Mo. Bot. Gard. 67: 469. (1980). झ Arachnothryx kirkbridei (Dwyer) Borhidi, Acta Bot. Acad. Sci. Hung. 28: 70. (1982).

5. Arachnothryx bourgaei (Standl.) Borhidi, Acta Bot. Hung. 28: 69. (1982). - Bas.: Rondeletia bourgaei Standl., N. Amer. Fl. 32: 56. (1918). - Tipo: México, Veracruz, Región de Orizaba, Monte San Cristóbal, Col.: E. Bourgeau (2618), 8. Jul. 1866; holotipo US 49964 n.v.; foto: MEXU! isotipo: P!, foto: MEXU!. - Distr.: México: Oaxaca, Veracruz, endémica.

= Rondeletia laniflora Hemsl., Biol. Centr. Amer. Bot. 2: 20. (1881) p.p., non Rondeletia laniflora Benth. (1841).

6. Arachnothryx brachythyrsa Borhidi, Acta Bot. Hung. 48: 39. (2006). - Tipo: México; Oaxaca; Mpio. San Jerónimo Coatlán, Distr. Miahuatlán; $41 \mathrm{~km}$ al SW de San Jerónimo Coatlán, brecha a Progreso. Col.: Alvaro Campos V. (3255); 7 Ago 1990; holotipo: MEXU!. - Distr.: México: Oaxaca, endémica.

7. Arachnothryx brenesii (Standl.) Borhidi, Acta Bot. Hung. 33: 301. (1987). - Bas.: Rondeletia brenesii Standl., Field Mus. Publ. Bot. 18: 1370. (1938). - Holotipo: Costa Rica. Col.: Brenes (6757) (F). - Ilustr.: Burger y Taylor, Fieldiana Bot. n.s. 33: 35. t. 21. (1993). Distr.: Costa Rica, endémica.

= Gonzalagunia exaltata Standl.

8. Arachnothryx breviflora Borhidi, Acta Bot. Hung. 46: 106. (2004). - Tipo: Mexico: Chiapas, Mpio. Pantepec: slope with montane rain forest above Rayón along road to 
Pantepec and Tapalapa. Elev.: 1770 m. Col.: D. E. Breedlove (52978), 22 Sep 1981; holotipo: MEXU! - Distr.: México: Chiapas, endémica. Incluída incorrectamente en la sinonimía del Arachnothryx buddleioides por D. Lorence.

9. Arachnothryx buddleioides (Benth.) Planch., Fl. Serres 5: tab. 442. (1849). (Fig. 5) Bas.: Rondeletia buddleioides Benth., Pl. Hartw. 69. (1840). - Tipo: México: Oaxaca, in planitie Llano Verde. Col.: T. Hartweg (502); holotipo: K!, foto: PTBG; isotipos: B, destruído, foto: MO!, K!, foto: NY!, fragmento US, foto: MEXU!, P!, foto: MEXU! - Distr.: México (Colima, Guerrero, México, Michoacán, Nayarit, Oaxaca, Tabasco Veracruz) y América Central hasta Panamá.

= Rondeletia affinis Hemsl., Diagn. Pl. Mex. 28. (1879).

= Rondeletia rothschuhii Loes. Engl. Bot. Jahrb. 60: 396. (1926).

= Arachnothryx longipetiolata (Lundell) Borhidi, Acta Bot. Hung. 35: 310. (1989). झ Rondeletia longipetiolata Lundell, Wrightia 5: 325. (1976).

= Arachnothryx pumae Torr.-Montúfar et H. Ochot., Rev. Mex. Biodiv. 84: 1082. (2013). - Los caracteres dados en la descripción y por el dibujo de Arachnothryx pumae todos son diferencias cuantitativas de los órganos vegetativo y muestran claramente, que se trata de una forma local, de Arachnothryx buddleioides en la area de Los Tuxtlas. Así Arachnothryx pumae cabe cómodamente a la variación riquísima del Arachnothryx buddleioides, con una distribución desde Colima hasta Panamá. Es muy difícil describir plantas nuevas sin conocer la flora Rubiácea de México.

10. Arachnothryx calycophylla Steyerm., Mem. NY. Bot. Gard. 17: 257. (1967). - Tipo: Venezuela; Tachira: between Las Delicias y Paraguita, bordering Rio Tachira along the Clombian-Venezuelan border, alt. 1675-1980 m. Col.: J. A. Steyermark (57136), 12 Jul 1944; holotipo: VEN. - Distr.: Colombia and Venezuela.

11. Arachnothryx calycosa (Donn. Sm.) Borhidi, Acta Bot. Acad. Sci. Hung. 28: 70. (1982). - Bas.: Rondeletia calycosa Donn. Sm., Bot. Gaz. 56: 59. (1913). - Holotipo: Costa Rica. Col.: Tonduz (11633) (US). - Ilustr.: Burger y Taylor, Fieldiana Bot. n.s. 33: 55. t. 41. (1993). - Distr.: Costa Rica, endémica.

12. Arachnothryx capitellata (Hemsl.) Borhidi, Acta Bot. Hung. 28: 69. (1982). - Bas.: Rondeletia capitellata Hemsl., Diagn. Pl. Mex. 28. (1879). - Tipo: México, sin localidad precisa. Col.: Jurgensen (355); lectotipo: K!, foto: PTBG n.v., isolectotipos: G-DEL n.v., fragmento F, n.v., K! - Distr.: México: Chiapas, Guerrero, Hidalgo, Oaxaca, Puebla, Veracruz; Guatemala.

12a. Arachnothryx capitellata subsp. capitellata

= Rondeletia liebmannii Standl., J. Wash. Acad. Sci. 17: 336. (1927). झ Arachnothryx liebmannii (Standl.) Borhidi, Acta Bot. Hung. 33: 302. (1987). - Distr.: México: Chiapas Guerrero, Oaxaca, Sur de Veracruz; Guatemala.

12b. Arachnothryx capitellata subsp. pringlei (Lorence) Borhidi, Acta Bot. Hung. 47: 29. (2005). - Bas.: Rondeletia pringlei Lorence, Novon 7: 54. (1997). $\equiv$ Arachnothryx pringlei (Lorence) Borhidi, Acta Bot. Hung. 40: 16. 1996-97 (publ. 1999). - Tipo: México: Veracruz... hills near Orizaba, 5000 ft. Col.: C. G. Pringle (6135), 9. Feb. 1895; holotipo: GH n.v., isotipos: K n.v., MEXU!, MO!, WU n.v. - Distr.: México: Hidalgo, Puebla, Veracruz, endémica.

= Rondeletia capitellata Standl., N. Amer. Fl. 32: 00. (1918). non Hemsl. (1879).

13. Arachnothryx caucana (Standl. et Steyerm.) Steyerm., Mem. NY. Bot. Gard. 17: 260. (1967). - Bas.: Rondeletia caucana Standl. et Steyerm., Acta Biol. Ven. 4: 108. (1964). - Tipo: Colombia; Cordillera Occidental: Dept. El Cauca, Rio Ingutto. Col.: E. Dryander (2094), Feb 1938. - Distr.: Colombia, endémica. 
14. Arachnothryx chaconii (Lorence) Borhidi, Acta Bot. Hung. 38: 140. 1993-94 (1995). - Bas.: Rondeletia chaconii Lorence, Fieldiana Bot. n.s. 33: 296. (1993). - Holotipo: Costa Rica. Col.: Chacón (177) (CR). - Ilustr.: Burger y Taylor, Fieldiana Bot. n.s. 33: 56. t. 41. (1993). Distr.: Costa Rica, endémica.

15. Arachnothryx chiapensis (Brand.) Borhidi, Acta Bot. Hung. 43: 42. (2001). - Bas.: Otocalyx chiapensis Brand., Univ. Calif. Publ. Bot. 6: 68. (1914). - Tipo: México, Chiapas, Cerro del Boquerón. Col.: C. A. Purpus (7041), 1913; holotipo UC 173047 n.v., fotos: MEXU!, isotipo: GH n.v. - Distr.: México: Chiapas, endémica.

= Rondeletia zolleriana Standl. et Steyerm., Publ. Field Mus. Nat. Hist. Bot. Ser. 22: 292. (1940). $\equiv$ Arachnothryx zolleriana (Standl. et Steyerm.) Borhidi, Acta Bot. Hung. 35: 311. (1989).

$=$ Rondeletia cordovana Standl. et Steyerm., Publ. Field Mus. Nat. Hist. Bot. Ser. 23: 26. (1943). $\equiv$ Arachnothryx cordovana (Standl. et Steyerm.) Borhidi, Acta Bot. Hung. 33: 301. (1987).

= Rondeletia brandegeeana Lorence, Novon 1: 138. (1991). nom. superfl.

16. Arachnothryx chimalaparum Lorence ex Borhidi, Rub. Méx. 45. (2006). - Tipo: México, Oaxaca, mpio. de Santa María Chimalapa. Col.: Wendt et al. (5676); holotipo: MO, isotipo: MEXU! - Distr.: México: Oaxaca, endémica.

17. Arachnothryx chimboracensis (Standl.) Steyerm., Mem. NY. Bot. Gard. 17: 258. (1967). - Bas.: Rondeletia chimboracensis Standl., Field Mus. Publ. Bot. 7: 203. (1931). - Tipo: Ecuador; Imbabura: Cordillera Occidental above Rio Chalguayaco below Magnolia, éower Intag Valley, alt. 1210 m. Col.: Drew (E-649). - Distr.: Ecuador, endémica.

18. Arachnothryx chinajensis (Standl. et Steyerm.) Borhidi, Acta Bot. Hung. 33: 301. (1987). - Bas.: Rondeletia chinajensis Standl. et Steyerm., Publ. Field Mus. Nat. Hist. Bot. Ser. 23: 254. (1947). - Tipo: Guatemala: Alta Verapaz, Cerro Chinajá, between Finca Yalpemech and Chinajá, above source of Rio San Diego, 150-700 m. Col.: J. A. Steyermark (45637), 1-2 Apr 1942; holotipo: F 1134210, n.v., foto: MEXU! - Distr.: México: Chiapas; Guatemala.

19. Arachnothryx chiriquiana (Lorence) Borhidi, Acta Bot. Hung. 40: 15. (1997). - Bas.: Rondeletia chiriquiana Lorence, Novon 4: 129. (1994). - Holotipo: Panamá. Col.: Churchill (5924) (MO). - Ilustr. Novon 4: 131. t. 7. (1994). - Distr.: Panamá, endémica.

20. Arachnothryx colombiana (Rusby) Steyerm., Mem. NY. Bot. Gard. 17: 258. (1967). - Bas.: Rondeletia colombiana Rusby, Descr. N. Sp. S. Am. Pl. 129. (1920). - Tipo: Colombia; Sierra de Onaca, alt. 1500 m. Col.: Herbert H. Smith (1813 in part), 22 Aug 1898; holotipo: NY. - Distr.: Colombia, endémica.

21. Arachnothryx costanensis Steyerm., Mem. NY. Bot. Gard. 17: 259. (1967). - Tipo: Venezuela; Anzoátegui: Cerro Peonía (Cerro Coroy) above “Carmelita," between Rio León and Bergantín, NE of Bergantín, alt. 1000-1450 m. Col.: J. A. Steyermark (61424), 7 Mar 1945; holotipo: VEN. - Distr.: Venezuela, endémica.

22. Arachnothryx cupreifolia (K. Schum. et K. Krause) Steyerm., Mem. NY. Bot. Gard. 17: 258. (1967). - Bas.: Rondeletia cupreifolia K. Schum. et K. Krause, Bot. Jahrb. 40: 314. (1908). - Tipo: Colombia; Antioquia, alt. 1700-2000 m. Col.: Lehmann (7289); holotipo: B十. - Distr.: Colombia y Ecuador.

23. Arachnothryx darienensis (Standl.) Borhidi, Acta Bot. Acad. Sci. Hung. 28: 68. (1982). - Bas.: Rondeletia darienensis Standl., N. Amer. Fl. 32: 53. (1918). - Holotipo: Panamá. Col.: Pittier (5684) (US). - Distr.: Mesoamérica, Colombia.

24. Arachnothryx discolor (HBK) Planch., Fl. Serres 5: sub plate 442. (1849). - Bas.: Rondeletia discolor HBK, Nov. Gen. Spec. 3: 396. (1820). - Tipo: Colombia... between Mariquita and Honda. Col.: Bonpland. - Distr.: Colombia, endémica. 
25. Arachnothryx dwyeri (Lorence) Borhidi, Acta Bot. Hung 38:140. 1993-94 (1995). - Bas.: Rondeletia dwyeri Lorence, Novon 1: 139. (1991). - Holotipo: Panamá. Col.: Gentry (4262) (MO). - Ilustr.: Ann. Mo. Bot. Gard. 67: 99. t. 21. (1980). - Distr.: Panamá, endémica. $=$ Chomelia leucophylla Dwyer, non Rondeletia leucophylla Kunth.

26. Arachnothryx educta (Standl. et Steyerm.) Steyerm., Mem. NY. Bot. Gard. 17: 260. (1967). - Bas.: Rondeletia educta Standl. et Steyerm. Acta Biol. Ven. 4: 109. (1964). - Tipo: Colombia. - Distr.: Colombia y Ecuador.

27. Arachnothryx euryphylla (Standl.) Steyerm., Mem. NY. Bot. Gard. 17: 258. (1967). - Bas.: Rondeletia euryphylla Standl., Field Mus. Publ. Bot. 7: 29. (1930). - Tipo: Colombia; Cordillera Occidental: Dept. Caldas, Belen, alt. 1500-1700 m. Col.: F. W. Pennell (10627), 15 Sep. 1922. - Distr.: Colombia, endémica.

28. Arachnothryx evansii (Lorence) Borhidi, Acta Bot. Hung. 40: 15. (1997). - Bas.: Rondeletia evansii Lorence, Novon 7: 52. (1997). - Holotipo: Honduras. Col.: House (1163) (PTBG). - Ilustr.: Lorence, Novon 7: 53. t. 4. (1997). - Distr.: Honduras, endémica.

29. Arachnothryx flocculosa Borhidi, Acta Bot. Hung. 45: 1. (2003). - Tipo: México: Chiapas, Mpio. Unión Juarez; 1 km al SO de Chiquihuite, camino a Unión Juárez, alt.: 1950 $\mathrm{m}$, bosque mesófilo de montaña. Col.: E. E. Martinez Soto y Abisai Garcia (22159), 18 Dec 1987; holotipo MEXU! - Distr.: México: Chiapas, endémica.

30. Arachnothryx fosbergii Steyerm., Mem. NY. Bot. Gard. 17: 255. (1967). - Tipo: Ecuador; Loja: headwaters of Rio Salado, $20 \mathrm{~km}$ NNW of Catamayo (La Toma). Col.: F. R. Fosberg y A. Giler (22964), 10 Feb. 1945; holotipo: NY, isotipo: US. - $\quad$ Distr.: Ecuador, endémica.

31. Arachnothryx garciae Standl. ex Steyerm., Mem. NY. Bot. Gard. 17: 257. (1967). - Tipo: Colombia; Cundinamarca: Cordillra Oriental, San Francisco, Finca "El Carmero", El Tablazo entre Subachoque y San Francisco, alt. 1900-2100 m. Col.: H. Garcia-Barriga (11037), 26 Jan 1944; holotipo: US. - Distr.: Colombia, endémica.

32. Arachnothryx ginetteae (Lorence) Borhidi, Acta Bot. Hung. 38: 140. 1993-94 (1995). - Bas.: Rondeletia ginetteae Lorence, Novon 1: 141. (1991). - Tipo: México: Oaxaca, Distr. de Tuxtepec, Ruta 175 Tuxtepec to Oaxaca, ca $10 \mathrm{~km} \mathrm{~S}$ of Valle Nacional, Sierra de Juárez, 750 m. Col.: D. H. Lorence y R. Cedillo T. (2973), 6 Dec 1980; holotipo: MEXU! - Distr.: México, Oaxaca, endémica. Incluída a la Flora Mesoamericana por D. Lorence, aunque, no existe en Mesoamérica.

33. Arachnothryx glabrata (Standl.) Steyerm., Mem. NY. Bot. Gard. 17: 261. (1967). Bas.: Rondeletia glabrata Standl., Field Mus. Publ. Bot. 7: 30. (1930). - Tipo: Colombia; Dept. Santander: between Piedecuesta and Las Vegas, alt. 2000-2500 m. Col.: E. P. Killip y A. C. Smith (15504), 19-24 Dec 1926. - Distr.: Colombia, endémica.

34. Arachnothryx gonzaleoides (Standl.) Borhidi, Acta Bot. Hung. 33: 302. (1987). Bas.: Rondeletia gonzaleoides Standl., Publ. Field Columb. Mus. Bot. Ser. 8: 160. (1930). Tipo: México, Chiapas, Pueblo Nuevo Solistahuacán, 2200 m. Col.: J. Linden (149), 1840; holotipo: LE n.v., fragmento: F n.v., MEXU!, isotipos: K!, P! foto: MEXU! - Distr.: México: Chiapas; Guatemala, Honduras.

35. Arachnothryx gracilis (Hemsl.) Borhidi, Acta Bot. Hung. 28: 69. (1982). - Bas.: Rondeletia gracilis Hemsl., Diagn. Pl. Nov. Mex. 53. (1880). - Tipo: Guatemala: Alta Verapaz, 3600 ft. Col.: H. von Tuerckheim (404), March 1879; holotipo K! foto: PTBG n.v. - Distr.: México (Chiapas) y Guatemala.

36. Arachnothryx gracilispica (Standl.) Borhidi, Acta Bot. Hung. 33: 302. (1987). Bas.: Rondeletia gracilispica Standl., Publ. Field Columb. Mus. Bot. Ser. 8: 342. (1931). - Tipo: México: Oaxaca, Sierra San Pedro Nolasco, Talea etc. Col.: C. Jurgensen (935), 1843-44; 
holotipo: K!, fotos: F, US n.v., fragmento F n.v., foto: MEXU! - Distr.: México: Veracruz y Oaxaca, endémica. Incluída a la Flora Mesoamericana por D. Lorence, aunque, no existe en Mesoamérica.

= Rondeletia minor Lundell, Wrightia 5: 326. (1976). $\equiv$ Arachnothryx minor (Lundell) Borhidi, Acta Bot. Hung. 35: 310. (1989).

= Arachnothryx sooana Borhidi, Acta Bot. Hung. 45: 4. (2003).

37. Arachnothryx guerrerensis (Lorence) Borhidi, Acta Bot. Hung. 38: 139. (1995). Bas.: Rondeletia guerrerensis Lorence, Novon 1: 143. Fig. 8C, D. (1991). - Tipo: México: Guerrero, Mun. Atoyac de Alvarez, a $23.5 \mathrm{~km}$ al NE el Paraiso, camino Atoyac-Puerto del Gallo, 1700 m. Col.: E. Martinez Soto y F. Barrie (5596), 22 Nov 1983; holotipo: MEXU!, isotipos: BM n.v., CAS n.v., CHAPA n.v., F n.v., MO! - Distr.: México: Guerrero, endémica.

38. Arachnothryx guettardioides Standl. et Steyerm., Mem. NY. Bot. Gard. 17: 260. (1967). - Tipo: Colombia; Forest on Quebrada Congo, Rio Chigorodo, 10 km E of Chigodora, 40 km s of Turbo, alt. 120 m. Col.: Oscar Haught (4712), 25 May 1945. - Distr.: Colombia, endémica.

39. Arachnothryx heteranthera (Brand.) Borhidi, Acta Bot. Hung. 28: 69. (1982). Bas.: Rondeletia heteranthera Brand., Univ. Calif. Publ. Bot. 4: 387. (1913). - Tipo: México: Veracruz, Baños del Carrizal. Col.: C. A. Purpus (6184), Aug 1912; holotipo: UC 155217, n.v., isotipos: F n.v., MO! - Distr.: México: Hidalgo, Oaxaca, Puebla, Querétaro, San Luis Potosí, Veracruz, endémica.

$=$ Rondeletia falciformis Lundell, Wrightia 5: 324. (1976). $\equiv$ Arachnothryx falciformis (Lundell) Borhidi, Acta Bot. Hung. 35: 310. (1989).

40. Arachnothryx hirtinervis Borhidi, Acta Bot. Hung. 45: 3. Fig. 1. (2003). - Tipo: México, Oaxaca, Distr. Villa Alta, Mpio. San Bartolomé Zoogocho, 9.7 km de Maravillas por la carretera Maravillas-Juquila, laderas de los Siete Picachos. Alt.: 2115 m. Col.: X. Munn, R. Aguilar, R. de Castillo y R. Rivera (159), 28 Oct 1996; holotipo: MEXU! - Distr.: México: Oaxaca, endémica. Incluída en la sinonimía de Arachnothryx capitellata (Hemsl.) Borhidi, por D. Lorence.

41. Arachnothryx hispidula Griseb., Fl. Brit. W. Ind. 325. (1864). - Tipo: Trinidad: Maraccas Waterfall. 10 Nov 1845. without collector indicated; holotipo: GOET, isotipo: NY. - Distr.: Trinidad, endémica.

$=$ Rondeletia hispidula (Griseb.) Schum. in Engler y Prantl, Nat. Pflanzenfam. 4(4): 35. (1891).

42. Arachnothryx jaliscensis Borhidi et E.Martínez, Acta Bot. Hung. 55: 223. (2013). Tipo: México, Jalisco, Mpio.: San Cristóbal de la Barranca. Arroyo Los Cuartos (El Escalón), $2 \mathrm{~km}$ al Oeste de La Arena por el camino a Los Pueblitos. $20^{\circ} 59^{\prime} 50^{\prime \prime} \mathrm{N}, 103^{\circ} 25^{\prime} 20^{\prime \prime} \mathrm{W}$, alt.: 1300 msnm. Bosque tropical caducifolio. Col.: P. Carrillo-Reyes, E. M. Barba y M. Ayón (443), 30 Aug 1998; holotipo: MEXU. - Ilustr.: Acta Bot. Hung. 55: 225. (2013). - Distr: México: Jalisco, endémica.

43. Arachnothryx jurgensenii (Hemsl.) Borhidi, Acta Bot. Hung. 28: 70. (1982). - Bas.: Rondeletia jurgensenii Hemsl., Diagn. Pl. Nov. Mex. 29. (1879). - Tipo: México sin localidad precisa, según el protologo Oaxaca, Sierra San Pedro Nolasco. Col.: Jurgensen (248); holotipo: K!, foto: PTBG n.v., isotipo G-DEL n.v. - Distr.: México: Chiapas, Nayarit, Oaxaca, Sinaloa; Guatemala, El Salvador.

= Stylosiphonia salvadorensis Standl., J. Wash. Acad. Sci. 14: 246. (1924).

= Deppea macrocarpa Standl., Publ. Field Columb. Mus. Bot. Ser. 4: 268. (1929).

= Deppea stenosepala Standl., Publ. Field Columb. Mus. Bot. Ser. 8: 161. (1930).

= Javorkaea pulcherrima Borhidi, Acta Bot. Hung. 45: 8. Fig. 2. (2003). 
44. Arachnothryx laniflora (Benth.) Planch., Fl. Serres 5: tab. 442. (1849). - Bas.: Rondeletia laniflora Benth., Pl. Hartw. 85. (1841). - Tipo: Guatemala: Mountains of Las Nubes. Col.: T. Hartweg (584), March 1841; holotipo: K!, foto: PTBG n.v., fragmento US n.v., foto: MEXU!, isotipos: B destruido, fotos: F n.v., MO!, US n.v., G-DEL n.v., fragmento: F n.v., P! foto: MEXU!; W!, foto: F n.v. - Distr.: México: Chiapas, Guerrero, Oaxaca; Guatemala, El Salvador.

$=$ Rondeletia albida Lundell, Wrightia 5: 323. (1976). $\equiv$ Arachnothryx albida (Lundell) Borhidi, Acta Bot. Hung. 35: 309. 1993-94 (1995).

45. Arachnothryx latiloba Borhidi, Acta Bot. Hung. 46: 56. (2004). - Tipo: México: Guerrero, $\pm 14 \mathrm{~km}$ al SSO del Campamento El Gallo, sobre el camino de Atoyac; estribaciones SO del Teotepec, alt. 1900 m. Col.: J. Rzedowski y McVaugh (89), 26 Jan 1965; holotipo: ENCB!; isotipo: MICH. - Distr.: México: Guerrero, endémica.

46. Arachnothryx leucophylla (Kunth) Planch., Fl. Serres 5: tab. 442. (1849). (Fig. 6.) - Bas.: Rondeletia leucophylla Kunth, in HBK Nov. Gen. Sp. 3: 395. (1820). - Tipo: México, Guerrero inter Alto del Peregrino et flumen Papagallo. Col.: Humboldt y Bonpland s.n.; holotipo: P-Bonpland n.v., isotipos: B destruido, fotos: F n.v., MO!; P! foto: MEXU! - Distr.: México: (Chiapas, Colima, Durango, Guerrero, Jalisco, México, Michoacán, Morelos, Nayarit, Oaxaca, Sinaloa, Veracruz) endémica.

= Rondeletia elongata Bartl. ex DC., Prodr. 4: 409. (1830).

= Bouvardia discolor Hook. et Arn., Bot. Beechey Voy. 428. (1840), non Rondeletia discolor Kunth, in Humb. y Bonpl. (1820).

= Rondeletia dubia Hemsl., Diagn. Pl. Mex. 28. (1879).

= Rondeletia leucophylla var. calycosa Greenm., Proc. Amer. Acad. Arts 39: 92. (1903).

$=$ Rondeletia leptodictya B. L. Robins., Proc. Amer. Acad. Arts 45: 402. (1910). $\equiv$ Arachnothryx leptodictya (B. L. Robins.) Borhidi, Acta Bot. Hung. 28: 68. (1982).

= Rondeletia yucatanensis Lundell, Wrightia 5: 329. (1976). $\equiv$ Arachnothryx yucatanensis (Lundell) Borhidi, Acta Bot. Hung. 35: 311. (1989).

47. Arachnothryx lineolata Borhidi, Acta Bot. Hung. 47. 26. (2005). - Tipo: México: Chiapas; la comunidad Lacandona de Nahá, Mpio. Ocosingo, $27 \mathrm{~km}$ al SE de Palenque por la carretera fronteriza hasta el crucero Chancalá, después $55.6 \mathrm{~km}$ por el camino de la

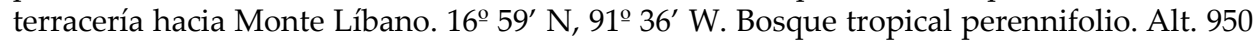
m. Col.: A. Durán F. y S. Levy T. (369), 20 Abril 1995; holotipo: MEXU! - Distr.: México: Chiapas, endémica. Erroneamente no incluída en la Flora Mesoamericana por D. Lorence.

48. Arachnothryx linguiformis (Hemsl.) Borhidi, Acta Bot. Hung. 28: 70. (1982). Bas.: Rondeletia linguiformis Hemsl., Diagn. Pl. Nov. Mex. 29. (1879). - Tipo: Guatemala: sin localidad precisa. Col.: Skinner (s.n.), 1857; holotipo: K!, foto: PTBG n.v. - Distr.: México : Chiapas, Guatemala.

= Rondeletia aetheocalymna Donn.-Sm., Bot. Gaz. (Crawfordsville) 42: 298. (1906). $\equiv$ Arachnothryx aetheocalymna (Donn.-Sm.) Borhidi, Acta Bot. Hung. 28: 70. (1982).

49. Arachnothryx linguiloba Borhidi et N. Diego-Pérez, Acta Bot. Hung. 51: 11. (2009). - Tipo: México: Guerrero; Mpio. Atoyac. Loc. El Ranchito, 13 km al NE de El Paraíso. Elev. 1100 m snm. Bosque mesófilo de montaña. Col.: José C. Soto Nuñez (11756), 14. Dic. 1985; holotipo: MEXU. - Distr.: México: Guerrero, endémica.

50. Arachnothryx lojensis Steyerm., Mem. NY. Bot. Gard. 17: 255. (1967). - Tipo: Ecuador; Loja: ridge between two main forks of Rio San Francisco, eastern slope of Cordillera de Zamora (El Condor) 12-15 km ENE of Loja; lat. $3^{\circ} 57-59^{\prime}$ S, long. 78 59-79’ W, Col.: R. F. Fosberg y M. A. Giler (23141); holotipo: NY. - Distr.: Ecuador, endémica. 
51. Arachnothryx macrocalyx (Standl. et Steyerm.) Borhidi, Acta Bot. Hung. 33: 302. (1987). - Bas.: Rondeletia macrocalyx Standl. et Steyerm., Publ. Field Mus. Nat. Hist. Bot. Ser. 23: 254. (1947). झ Javorkaea macrocalyx (Standl. et Steyerm.) Borhidi, Acta Bot. Hung. 43: 50. (2001). - Tipo: Guatemala: Izabal, Cerro San Gil, 300-900 m. Col.: J. A. Steyermark (41864), 25 Dec 1941; holotipo: F 1134224 n.v., foto: MEXU!, isotipo: F n.v., foto: MEXU!, US n.v. Distr.: México: Chiapas; Guatemala.

52. Arachnothryx manantlanensis (Lorence) Borhidi, Acta Bot. Hung. 38: 139. 199394 (1995). - Bas.: Rondeletia manantlanensis Lorence, Novon 1: 145. Fig. 4A, B. (1991). - Tipo: México: Jalisco, Mun. de Autlán, Cañada del Laurelito, La Joyas, 1850 m. Col.: A. Vázquez (3750), 7 Dec 1985; holotipo: WIS n.v. foto: PTBG n.v.; isotipo: IBUG n.v. - Distr.: México: Jalisco, endémica.

53. Arachnothryx megalantha (Lorence) Lorence, Novon 15: 451. (2005). - Bas.: Rondeletia megalantha Lorence, Novon 4: 132. (1994). - Holotipo: Honduras. Col.: Carleton (653) (US). - Ilustr.: Lorence, Novon 4: 133. t. 8. (1994). - Distr.: Honduras, endémica.

54. Arachnothryx mexicana (Turcz.) Borhidi, Acta Bot. Hung. 43: 51. (2001). - Bas.: Siphonandra mexicana Turcz., Bull. Soc. Imp. Naturalistes Moscou 21: 581. (1848). $\equiv$ Rondeletia mexicana (Turcz.) Standl., Publ. Field Mus. Nat. Hist. Bot. Ser. 22: 388. (1940). - Tipo: México: Oaxaca, sin localidad precisa, $3000 \mathrm{ft}$. Col.: H. Galeotti (2664bis), Oct.; holotipo: G-DEL! fotos: F n.v., MO! US n.v.; isotipo: P!, foto: MEXU!; fragmento F n.v. fotos: MEXU! - Distr.: México, Oaxaca, endémica.

$=$ Rondeletia polycephala Standl., J. Wash. Acad. Sci. 17: 337. (1927). $\equiv$ Arachnothryx polycephala (Stand1.) Borhidi, Acta Bot. Hung. 35: 310. (1987).

$=$ Rondeletia oaxacana Standl., Field Mus. Nat. Hist. Bot. Ser. 11: 255. (1936). झ Arachnothryx oaxacana (Standl.) Borhidi, Acta Bot. Hung. 33: 302. (1987).

55. Arachnothryx michoacana Borhidi, Acta Bot. Hung. 46. 57. (2004). - Tipo: México: Michoacán, 12.8 miles N of Anguililla on road to Dos Aguas: pine-oak woods; elevation 1570 m. Col.: Melinda F. Denton (2016), 7 Aug 1972; holotipo: ENCB!, isotipo: Univ. of Washington. - Distr.: México: Michoacán, endémica.

56. Arachnothryx monteverdensis (Lorence) Borhidi, Acta Bot. Hung. 38: 140. 199394 (1995). - Bas.: Rondeletia monteverdensis Lorence, Novon 1: 147. (1991). - Holotipo: Costa Rica. Col.: Haber (2340) (MO). - Ilustr.: Lorence, Novon 1: 147. t. 5A B, (1991). - Distr.: Costa Rica, endémica.

57. Arachnothryx monticola Borhidi, Acta Bot. Hung. 46: 59. (2004). - Tipo: México: Guerrero, Mun. Atoyac de Alvarez, $16 \mathrm{~km}$ sobre el camino de Puente del Gallo a Atoyac, alt. 1950 m. Col.: M. E. Millan (175), Mar 1983; holotipo: FCME!, isotipo: ENCB! - Distr.: México: Guerrero, endémica.

58. Arachnothryx myriantha (Standl. et Steyerm.) Borhidi, Acta Bot. Hung. 33: 302. (1987). - Bas.: Rondeletia myriantha Standl. et Steyerm., Field Mus. Publ. Bot. 22: 288. (1940). - Holotipo: Guatemala. Col.: Skutch (1569) (F). - Distr.: Guatemala, endémica.

59. Arachnothryx nebulosa (Standl.) Borhidi, Acta Bot. Hung. 33: 302. (1987). - Bas.: Rondeletia nebulosa Standl., Trop. Woods 37: 32. (1934). - Holotipo: Honduras. Col.: Edwards (P10) (F). - Distr.: Honduras, endémica.

60. Arachnothryx nelsonii Lorence, Novon 15: 448. (2005). - Holotipo: Honduras. Col.: Hawkins (993) (MO). - Ilustr.: Lorence, Novon 15: 449. t. 1. (2005). - Distr.: Honduras, endémica.

61. Arachnothryx nitida (Hemsl.) Borhidi, Acta Bot. Hung. 28: 69. (1982). - Rondeletia nitida Hemsl., Diagn. Pl. Nov. Mex. 29. (1879). - Tipo: México: Chiapas, fl. Rosatres forets 
des pins, en fevr. Col.: Linden (1660); lectotipo K!, foto: PTBG n.v., isolectotipos: G-DEL n.v. P!, foto: MEXU! - Distr.: México: Oaxaca y Chiapas, endémica.

62. Arachnothryx ovandensis (Lundell) Borhidi, Acta Bot. Hung. 35: 310. (1989). Bas.: Rondeletia ovandensis Lundell, Wrightia 5: 326. (1975). - Tipo: México: Chiapas, Mt. Ovando, near Escuintla. Col.: E. Matuda (6067), 24 Oct 1941; holotipo: TEX-LL n.v., foto: MEXU!, isotipos: F n.v., MEXU!, TEX-LL n.v. - Distr.: México Chiapas, Guatemala. Está tratada en la Flora Mesoamérica como Arachnothryx armetalis (L. O. Wms.) Lorence, que es un sinónimo posterior.

= Rondeletia myriantha Standl. et Steyerm. var. armentalis L. O. Wms., Phytologia 26: 127. (1973). $\equiv$ Arachnothryx myriantha (Standl. et Steyerm.) Borhidi var. armentalis (L. O. Wms.) Borhidi, Acta Bot. Hung. 35: 310. (1989). = Arachnothryx armentalis (L. O. Wms.) Lorence, Novon, 12: 00. (2005).

63. Arachnothryx ovata (Rusby) Steyerm., Mem. NY. Bot. Gard. 17: 259. (1967). - Bas.: Rondeletia ovata Rusby, N. Sp. S. Am. Pl. 129. (1929). - Tipo: Colombia; Stream at Las Nubes, Santa Marta, alt. 1365 m. Col.: Herbert H. Smith (1813 in part), 16 Dec 1898; holotipo: NY. - Distr.: Colombia, endémica.

64. Arachnothríyx pansamalana (Standl.) Borhidi, Acta Bot. Acad. Sci. Hung. 28: 69. (1982). - Bas.: Rondeletia pansamalana Standl., N. Amer. Fl. 32: 58. (1918). - Holotipo: Guatemala. Col.: von Türckheim (897) (US). - Distr.: Guatemala, endémica.

65. Arachnothryx pauciflora Borhidi, Acta Bot. Hung. 47: 28. (2005). - Tipo: México:

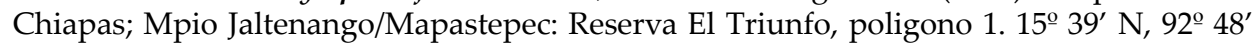
W, alt: 1600 m. Col.: M. Heath y A. Long (1007), 17 Jun 1990; holotipo: MEXU! - Distr.: México (Chiapas), endémica. Erroneamente no incluída en la Flora Mesoamericana por D. Lorence.

66. Arachnothryx perezii (Standl. et Steyerm.) Steyerm., Mem. NY. Bot. Gard. 17: 258. (1967). - Bas.: Rondeletia perezii Standl. et Steyerm., Acta Biol. Ven. 4: 111. Fig. 53. (1964). Distr.: Colombia, endémica.

67. Arachnothryx peruviana (Stand1.) Steyerm., Mem. NY. Bot. Gard. 17: 251. (1967). - Bas.: Rondeletia peruviana Standl., Field Mus. Publ. Bot. 8: 342. (1936). - Tipo: Peru; Dept. Loreto; Mouth of Rio Santiago Upper Marañon, alt. 160 m. Col.: Tessmann (4223). - Distr.: Peru, endémica.

68. Arachnothryx povedae (Lorence) Borhidi, Acta Bot. Hung. 38: 140. (1995). - Bas.: Rondeletia povedae Lorence, Fieldiana Bot. n.s. 33: 299. (1993). - Holotipo: Costa Rica. Col.: Poveda (441) (CR). - Ilustr.: Burger y Taylor, Fieldiana Bot. n.s. 33: 56 t. 41A (1993).

69. Arachnothryx purpurea (Lorence) Borhidi, Acta Bot. Hung. 38: 139. (1995). - Bas.: Rondeletia purpurea Lorence, Novon 1: 148. Figs 6A-F, 7A-B, (1991). - Tipo: México: Oaxaca, Distr. de Ixtlán, Sierra de Juárez, Ruta 175 Tuxtepec a Oaxaca, a $5 \mathrm{~km}$ al N de Vista Hermosa, 1175 m. Col.: D. H. Lorence y R. Torres (4009), 14 Apr 1982; holotipo: MEXU!, foto: PTBG n.v., isotipos: BM!, F n.v., MO! - Distr.: México: Oaxaca, endémica. Se encuentra en Mesoamérica según Lorence.

70. Arachnothryx pyramidalis (Lundell) Borhidi, Acta Bot. Hung. 35: 310. (1989). Bas.: Rondeletia pyramidalis Lundell, Wrightia, 5: 327. (1975). - Tipo: México: Chiapas, San Luis near Siltepec, 1300 m. Col.: E. Matuda (5292), 24 Jan 1945; holotipo: TEX-LL n.v., foto: MEXU!, isotipos: F n.v., MEXU!, TEX-LL n.v. - Distr.: México: Chiapas, Guerrero, Oaxaca; endémica. Se encuentra en Mesoamérica según Lorence.

71. Arachnothryx reflexa (Benth.) Planch., Fl. Serres 5: sub plate 442. (1849). - Bas.: Rondeletia reflexa Benth., Pl. Hartw. 192. (1845). - Tipo: Colombia; Near Villeta, Prov. Bogotá. Col.: Hartweg (1052). - Distr.: Colombia y Venezuela. 
71a. Arachnothryx reflexa var. reflexa

= Arachnothryx rosea Linden, Hort. Linden 2: 3. (1859). झ Rondeletia rosea (Linden) Standl., Field Mus. Publ. Bot. 7: 34. (1930).

71b. Arachnothryx reflexa var. inconstans (Standl.) Steyerm., Mem. NY. Bot. Gard. 17: 252. (1967). - Bas.: Rondeletia inconstans Standl., Field Mus. Publ. Bot. 7: 31. (1930). Tipo: Colombia, Qurtame Pipiral, Andes de Bogotá, alt. 1000-1600 m. Col.: F. C. Lehmann (8751), July 1897). - Distr.: Andes de Colombia.

= Rondeletia orthoneura K. Schum. et Kr., Bot. Jahrb. 40: 314. (1908).

= Rondeletia orthoneura var. angustior K. Schum. et Kr., Bot. Jahrb. 40: 314. (1908).

71c. Arachnothryx reflexa var. breviloba Steyerm., Mem. NY. Bot. Gard. 17: 253 (1967). - Tipo: Colombia; Norte de Santander, La Camarona, $6 \mathrm{~km}$ W of Ocaña, lat. $8^{\circ} 14^{\prime}$ N, long. $73^{\circ} 24^{\prime}$ W, alt. 1700-1800 m. Col.: F. R. Fosberg (21418), 14 Dec. 1943; holotipo: NY. - Distr.: Colombia and Venezuela.

71d. Arachnothryx reflexa var. glandulifera Steyerm., Mem. NY. Bot. Gard. 17: 253 (1967). - Tipo: Venezuela; Anzoátegui: along Rio Zumbador and tributary, near base of Piedra Blanca, NE of Bergantín, alt. 800-1000 m. Col.: J. A. Steyermark (61317), 1-2 March, 1945; holotipo: VEN. - Distr.: Venezuela.

71e. Arachnothryx reflexa var. meridensis Steyerm., Mem. NY. Bot. Gard. 17: 253. (1967). - Tipo: Mérida: between Los Corales and Las Cuadras, alt. 1490-3210 m. Col.: J. A. Steyermark (55775), 25 Mar. 1944; holotipo: VEN. - Distr. Venezuela.

71f. Arachnothryx reflexa var. calycina Steyerm., Mem. NY. Bot. Gard. 17: 255. (1967). - Tipo: Colombia; Magdalena: Sierra Nevada de Santa María, SE slopes, Hoya del Rio Donachuí, ravine SE od Donachuí, alt. 1350-1500 m. Col.: José Cuatrecasas y R. Romero Castaneda (24332), 22 Sep 1959; holotipo: VEN. - Distr.: Colombia.

71g. Arachnothryx reflexa var. trichotoma (Standl.) Steyerm., Mem. NY. Bot. Gard. 17: 255. (1967). - Bas.: Rondeletia trichotoma Standl., Field Mus. Publ. Bot. 7: 204. (1931). Tipo: Ecuador; Ades at the base of Mt. Chimborazo, alt. 900 m. Col.: R. Spruce (6187), July 1860. - Distr.: Ecuador.

72. Arachnothryx rekoi (Standl.) Borhidi, Acta Bot. Hung. 28: 69. (1982). - Bas.: Rondeletia rekoi Standl., Proc. Biol. Soc. Wash. 8: 126. (1918). - Tipo: México: Oaxaca, Las Pilas (Cerro Espino), 400 m. Col.: B. P. Reko (3490), 10 Oct 1917; holotipo: US 867145 n.v., foto: MEXU! - Distr.: México: Oaxaca, endémica.

73. Arachnothryx ricoae (Lorence) Borhidi, Acta Bot. Hung. 38: 139. (1995). - Bas.: Rondeletia ricoae Lorence, Novon 1: 152. Figs 8E-F, 9A-B. (1991). - Tipo: México: Oaxaca, Distr. de Putla de Guerrero, a $10 \mathrm{~km}$ al NE de Putla, $950 \mathrm{~m}$. Col.: L. Rico A., E. M. Martinez y O. Téllez V. (566), 9 Dec 1982; holotipo; MEXU!; isotipos: BM!, F n.v., MO! - Distr.: México: Oaxaca, endémica.

74. Arachnothryx rubens (L. O. Wms.) Borhidi, Acta Bot. Hung. 35: 311. (1989). Bas.: Rondeletia rubens L. O. Wms., Phytologia 26: 128. (1973). - Holotipo: Guatemala. Col.: Skutch (1725) (F). - Distr.: Guatemala, endémica.

75. Arachnothryx rufescens (B. L. Rob.) Borhidi, Acta Bot. Hung. 28: 69. (1982). - Bas.: Rondeletia rufescens B. L. Rob., Proc. Amer. Acad. Arts 45: 402. (1910). - Tipo: Guatemala: Alta Verapaz, Cobán, 4800 ft. Col.: H. von Tuerckheim (582), Mar 1881; holotipo: GH n.v.; isotipos: F n.v., K!, P!, foto: MEXU!, US n.v., foto: MEXU! - Distr.: México: Chiapas; Guatemala.

76. Arachnothryx rugulosa (Standl.) Steyerm., Mem. NY. Bot. Gard. 17: 258. (1967). Bas.: Rondeletia rugulosa Standl., Field Mus. Publ. Bot. 7: 34. (1930). - Tipo: Colombia (New Grenada). Col.: I. Linden., 1842-1843; holotipo: K. - Distr.: Colombia, endémica. 
77. Arachnothryx rzedowskii (Lorence) Borhidi, Acta Bot. Hung. 38: 139. (1995). Bas.: Rondeletia rzedowskii Lorence, Novon 1: 154. Fig. 10. (1991). - Tipo: México: Guerrero, Mun. De Atoyac, Puerto de la Piedra Acanalada, 1930 m. Col.: Laboratorio de Biogeografía (867), 6 Jan 1984; holotipo: MEXU 364217!, isotipos: FCME! MEXU! - Distr.: México, Guerrero, endémica.

78. Arachnothryx sanchezii Borhidi, Acta Bot. Hung. 52: 77. (2010). - Tipo: México. Oaxaca. Distr.: Tehuantepec, Mpio. San Pedro Huamelula. Arroyo El Potrero. Selva baja caducifolia con vegetación secundaria del arroyo. 16 $6^{\circ} 1^{\prime} 54.3^{\prime \prime} \mathrm{N} 95^{\circ} 42^{\prime} 52^{\prime \prime} \mathrm{W}$, alt.: $146 \mathrm{msnm}$. Col.: Arturo Sánchez Martinez (2602), con F. Maldonado y .Leyva, 7 Jun 2009; holotipo: MEXU, isotipos: FCME, SERO, MO, UJP. - Distr.: México: Oaxaca, endémica.

79. Arachnothryx scabra (Hemsl.) Borhidi, Acta Bot. Hung. 46: 115. (2004). - Bas.: Rondeletia scabra Hemsl., Diagn. Pl. Nov. 29. (1879). =Javorkaea scabra (Hemsl.) Borhidi, Acta Bot. Hung. 43: 38. (2001). - Tipo: México: Oaxaca, Sierra San Pedro Nolasco, Talea etc. Col.: C. Jurgensen (926); holotipo: K!, foto: PTBG n.v. - Distr.: México: Oaxaca, endémica. En Mesoamérica según Lorence.

= Sommera acuminata Oerst. ex Standl., J. Wash. Acad. Sci. 17: 339. (1927). इ Rondeletia acuminata (Oerst. ex Standl.) Lorence et Cast.-Campos, Biotica 13: 147. (1988). झ Arachnothryx acuminata (Oerst. ex Standl.) Borhidi, Acta Bot. Hung. 35: 310. (1989). इ Javorkaea acuminata (Oerst. ex Standl.) Borhidi, Acta Bot. Hung. 40: 18. (1999).

$=$ Rondeletia mexiae Standl., Publ. Field Mus. Nat. Hist. Bot. Ser. 22: 55. (1940). झ Arachnothryx mexiae (Standl.) Borhidi, Acta Bot. Hung. 33: 302. (1987).

80. Arachnothryx scoti (Lorence) Borhidi, Acta Bot. Hung. 38: 139. (1995). - Bas.: Rondeletia scoti Lorence, Novon 1: 155. Fig. 8A-B. (1991). - Tipo: México: Oaxaca, La GlorietaRio Grande N-slope. Col.: T. MacDougall (s.n.), 12 Mar 1953; holotipo: MEXU!; isotipos: MO!, PTBG n.v. - Distr.: México, Oaxaca, endémica. Esperada en Chiapas y Mesoamérica según Lorence.

81. Arachnothryx secunda (Standl.) Borhidi, Acta Bot. Acad. Sci. Hung. 28: 70. (1982). - Bas.: Rondeletia secunda Standl., Contr. U.S. Natl. Herb. 18: 141. (1916). - Holotipo: Panamá. Col.: Pittier (4279) (US). - Distr.: Panamá, endémica.

$=$ Rondeletia darcyi Dwyer $\equiv$ Arachnothryx darcyi (Dwyer) Borhidi

82. Arachnothryx secundiflora (B. L. Rob.) Borhidi, Acta Bot. Hung. 28: 69. (1982). - Bas.: Rondeletia secundiflora B. L. Rob., Proc. Amer. Acad. Arts 45: 403, (1910). - Tipo: Guatemala: In woods along road from Patín to Escuintla. Col.: S. Hayes (s.n.), 21 Jul 1860; holotipo: GH n.v., isotipo: K!, foto: PTBG. - Distr.: México (Chiapas, Oaxaca y Tabasco); Guatemala.

$=$ Rondeletia vulcanicola Standl. et Steyerm., Publ. Field Mus. Nat. Hist. Bot. Ser. 22: 291. (1940). $\equiv$ Arachnothryx vulcanicola (Standl. et Steyerm.) Borhidi, Acta Bot. Hung. 33: 303. (1987).

83. Arachnothryx septicidalis (B. L. Rob.) Borhidi, Acta Bot. Hung. 28: 69. (1982). - Bas.: Rondeletia septicidalis B. L. Rob., Proc. Amer. Acad. Arts 45: 403. (1910). - Tipo: México: Chiapas, Chicharras, 3000-6000 ft. Col.: E. W. Nelson (3755), 6 Feb 1896; holotipo: US 256722 n.v., foto: MEXU!, isotipo: GH n.v. - Distr.: México: Chiapas; Guatemala.

84. Arachnothryx sessilis Borhidi et G. Ortiz, Acta Bot. Hung. 48: 40. (2006). - Tipo: México; Tabasco, municipio Huimanguillo, km 16-23 carretera hacia la Antena de microondas, entronque con la carretera Estación Chontalpa aMalpaso. Col.: G. Ortiz G. (5155), 23 Ago 1997; holotipo: MEXU. - Distr.: México: Tabasco, endémica. No incluída a la Flora Mesoamericana por D. Lorence. 
85. Arachnothryx sinaloae Borhidi, Acta Bot. Hung. 46: 115. (2004). - Tipo: México: Sinaloa, Mpio. Cosalá, el Camichin a $3 \mathrm{~km}$ al poniente de Guadelupe de los Reyes, alt.: 500-700 m, bosque tropical caducifolio. Col.: Rito Vega, Germán Bojorquez y A. Hernandez (3488), 21 Sep. 1989; holotipo: MEXU. - Distr.: México: Sinaloa, endémica.

86. Arachnothryx skutchii (Standl. et Steyerm.) Borhidi, Acta Bot. Hung. 33: 302. (1987). - Bas.: Rondeletia skutchii Standl. et Steyerm., Field Mus. Publ. Bot. 33: 289. (1940). Holotipo: Guatemala. Col.: Skutch (2110) (F). - Distr.: Guatemala, endémica.

87. Arachnothryx sousae Borhidi, Acta Bot. Hung. 46: 117. (2004). - Tipo: México: Chiapas: Mpio. Unión Juárez: en el Volcán Tacaná a $500 \mathrm{~m}$ al E de Talquián. alt.: $1700 \mathrm{~m}$. Col.: E. Martinez S., A. Márquez, G. Urquijo y M. Ramirez (19755), 7 Feb 1987; holotipo: MEXU! isotipo: CHAPA. - Distr.: México: Chiapas, endémica.

88. Arachnothryx spectabilis (Steyerm.) Rova, Delprete et B. Bremer, Ann. Mo. Bot. Gard. 96: 192. (2009). - Bas.: Cuatrecasasiodendron spectabile Steyerm., Acta Biol. Venez. 4: 33. (1964). - Tipo: Colombia, Valle de Cauca; Dept. Costa del Pacífico, Rio Cajambre, Barco. alt. 5-80 m. Col.: J. Cuatrecasas (17165), 21-30 Apr. 1944; holotipo: US, isotipo: VEN. - Distr.: Colombia, endémica.

= Cuatrecasasiodendron colombianum Standl. et Steyerm., Acta Biol. Venez. 4: 30. (1964).

89. Arachnothryx stachyoidea (Donn.-Sm.) Borhidi, Acta Bot. Hung. 28: 69. (1982). - Bas.: Rondeletia stachyoidea Donn.-Sm., Bot. Gaz. (Crawfordsville) 42: 298. (1906). - Tipo: Guatemala: Alta Verapaz, Semococh, 600-900 m. Col.: R. Hay (s.n.), 25 Apr 1905; holotipo: US 473726 n.v., foto: MEXU! - Distr.: México: Chiapas, Oaxaca, Tabasco y Veracruz; Belize, Guatemala y Honduras.

90. Arachnothryx subglabra Borhidi et N. C. Jiménez, Acta Bot. Hung. 48: 41. (2006). - Tipo: México; Tabasco, Boca del Cerro, Tenosique. Col.: N. del C. Jimenez P. (665), 22 Jun 2002; holotipo: MEXU! - Distr.: México: Tabasco, endémica. No incluída en la Flora Mesoamericana por D. Lorence.

91. Arachnothryx tabascensis Borhidi, Acta Bot. Hung. 46: 119. (2004). - Tipo: México: Tabasco: Mpio. Huimanguillo, $16-23 \mathrm{~km}$ hacia la Antena de microondas, entronque con la carretera Estación Chontalpa-Malpaso. Col.: G. Ortiz G. (5160); holotipo: MEXU! Distr.: México: Tabasco, endémica. Incluída erroneamente en la sinonimia de Arachnothryx buddleioides (Benth.) Planch. por D. Lorence.

92. Arachnothryx tacanensis (Lundell) Borhidi, Acta Bot. Hung. 33: 303. (1987). Bas.: Rondeletia tacanensis Lundell, Bull. Torr. Bot. Club 66: 603. (1939). - Tipo: México: Chiapas, Volcán Tacaná West, 2800 m. Col.: E. Matuda (2928), 30 Mar 1939; holotipo: TEXLL n.v., foto: MEXU!; isotipos: A n.v., F n.v., foto: MEXU!; GH n.v., K!, MEXU!, US n.v., foto: MEXU! - Distr.: México: Chiapas; Guatemala.

93. Arachnothryx tayloriae (Lorence) Borhidi, Acta Bot. Hung. 38: 140. 1993-94 (1995). - Bas.: Rondeletia tayloriae Lorence, Fieldiana Bot. n.s. 33: 300. (1993). - Holotipo: Costa Rica. Col.: Herrera y Chacón (2656) (CR). - Ilustr.: Burger y Taylor, Fieldiana Bot. n.s. 33: 56. t. 41A. (1993). - Distr.: Costa Rica, endémica.

94. Arachnothryx tenorioi (Lorence) Borhidi, Acta Bot. Hung. 38: 139. (1995). - Bas.: Rondeletia tenorioi Lorence, Novon 1: 156. Fig. 11A-B. (1991). - Tipo: México: Oaxaca, Distr. Juxtlahuaca, $19 \mathrm{~km}$ al SW de Juxtlahuaca y a $5 \mathrm{~km}$ en la misma dirección de Sta. Ma. Asunción, 1900 m. Col.: P. Tenorio y R. Torres C. (184), 4 Apr 1982; holotipo: MEXU!, foto: PTBG; isotipos: BM!, F n.v., MO! - Distr.: México: Oaxaca, endémica.

95. Arachnothryx tenuisepala Borhidi, Acta Bot. Hung. 45: 6. (2003). - Tipo: México: Veracruz, Sierra de Santa Maria al N del Ocotal Grande, Soteapan, alt.: 1475 m, selva pri- 
maria perennifolia. Col.: J. H. Beamon (5426), 7 Jan 1972; holotipo: MEXU! - Distr.: México: Veracruz, endémica.

96. Arachnothryx thiemei (Donn. Sm.) Borhidi, Acta Bot. Acad. Sci. Hung. 28: 69. (1982). - Bas.: Rondeletia thiemei Donn. Sm., Bot. Gaz. 42: 299. (1906). - Holotipo: Honduras. Col.: Thieme (5276) (US). - Distr.: Honduras, endémica.

97. Arachnothryx torresii (Standl.) Borhidi, Acta Bot. Hung. 33: 303. (1987). - Bas.: Rondeletia torresii Standl., J. Wash. Acad. Sci. 18: 163. (1928). = Javorkaea torresii (Standl.) Borhidi. - Holotipo: Costa Rica. Col.: Standley y Torres (47839) (US). - Distr.: Costa Rica, endémica.

98. Arachnothryx tuxtlensis (Lorence et Castillo-Campos) Borhidi, Acta Bot. Hung. 35: 311. (1989). - Bas.: Rondeletia tuxtlensis Lorence et Castillo-Campos, Biotica 13: 148. Fig. 1. (1988). - Tipo: México: Veracruz, Mun. Mecayapan, Volcán San Martin Pajapan al S del Ejido La Valentina, 700 m. Col.: J. I. Calzada (11183), 8 May 1985; holotipo: XAL!; isotipos: F n.v., MEXU!, MO! - Distr.: México: Veracruz, endémica.

99. Arachnothryx uxpanapensis (Lorence et Castillo-Campos) Borhidi, Acta Bot. Hung. 35: 311. (1989). - Bas.: Rondeletia uxpanapensis Lorence et Castillo-Campos, Biotica 13: Fig. 2. 150. (1988). इ Javorkaea uxpanapensis (Lorence et Castillo-Campos) Borhidi, Acta Bot. Hung. 40: 19. (1999). - Tipo: México: Veracruz, Mun. Hidalgotitlán, zona de Uxpanapa; Rio Solosuchil a 5-6 km ESE de Hermanos Cedillo, 100-200 m. Col.: D. H. Lorence et al. (3909), 24 Mar 1982; holotipo: MEXU!, foto: PTBG; isotipos: BM n.v., ENCB!, CHAPA n.v., MO!, NY!, PTBG n.v., UC n.v., XAL! - Distr.: México: Oaxaca y Vercruz, endémica. No se encuentra pero esperada su existencia en Mesoamérica según Lorence.

100. Arachnothryx venezuelensis Steyerm., Mem. NY. Bot. Gard. 17: 256. (1967). Tipo: Venezuela; Aragua: carretera a Choroni, alt 1300 m. Col.: C. E.Chardón (179), 26 Oct. 1940; holotipo: VEN. - Distr.: Venezuela, endémica.

101. Arachnothryx villosa (Hemsl.) Borhidi, Acta Bot. Hung. 28: 69. (1982). - Bas.: Rondeletia villosa Hemsl., Diagn. Pl. Nov. 27. (1879). - Tipo: México: Oaxaca, San Pedro Nolasco etc. Col.: C. Jurgensen (806); holotipo: K!, foto: PTBG n.v., isotipos: G-DEL n.v., K!, P!, fotos: MEXU!, PTBG. - Distr.: México: Oaxaca, Tabasco y Chiapas endémica.

102. Arachnothryx wendtii (Lorence et Castillo-Campos) Borhidi, Acta Bot. Hung. 35: 311. (1989). - Bas.: Rondeletia wendtii Lorence et Castillo-Campos, Biotica 13: 154. Fig. 3. (1988). - Tipo: México: Oaxaca, Distr. Juchitán, Mun. de Santa Maria Chimalapa, Arroyo Chocolín, Población de Nicolás Bravo, cerca del Rancho de Agustín Montero, 3-4 km al S de la población de Río Alegre, $150 \mathrm{~m}$. Col.: T. Wendt, A. Montero y I Almaraz (4244), 21 Oct 1983; holotipo: MEXU!, foto: PTBG, isotipos: CHAPA n.v., PTBG n.v. - Distr.: México: Oaxaca y Veracruz, endémica. No se encuentra pero esperada su existencia en Mesoamérica según Lorence.

\section{ESPECIES EXCLUÍDAS}

Arachnothryx costaricensis (Standl.) Borhidi, Acta Bot. Acad Sci. Hung. 28: 70. (1982). - Basónimo: Rondeletia costaricensis Standl., N. Amer. Fl. 32: 61. (1918). - Holotipo: Costa Rica. Col.: Pittier (16696) (US). - Ilustr.: Burger y Taylor, Fieldiana Bot. n.s. 33: 55. t. 41. (1993). = Renistipula costaricensis (Standl.) Borhidi, Acta Bot. Hung. 46: 123. (2004).

Arachnothryx deamii (Donn. Sm.) Borhidi, Acta Bot. Acad. Sci. Hung. 28: 70. (1982). Basónimo: Bouvardia deamii Donn. Sm., Bot. Gaz. 49: 455. (1910). Rondeletia deamii Standl., N. Amer. Fl. 32: 60. (1918). - Holotipo: Guatemala. Col.: Deam (6190) (US). = Donnelly- 
anthus deamii (Donn. Sm.) Borhidi, Acta Bot. Hung. 53: 278. (2011). - Según los estudios moleculares el género Donnellyanthus (tratado como Rondeletia deamii) pertenece a la tribu Rondeletieae s. str. mientras el género Arachnothryx pertenece a la tribu Guettardeae (Rova et al. 2009, Mans y Bremer 2010).

Arachnothryx galeottii (Standl.) Borhidi, Acta Bot. Acad. Sci. Hung. 28: 70. (1982). Basónimo: Rondeletia galeottii Standl., N. Amer. Fl. 32: 59: (1918). - Holotipo: México, estado desconocido. Col.: Galeotti (sine num.) (US) = Renistipula galeottii (Standl.) Borhidi, Acta Bot. Hung. 46: 123. (2004). - Ilustr.: Borhidi, Rub. Mex. 477. Fig. 104. (2006).

Arachnothryx hondurensis (Donn. Sm.) Lorence, Novon 15: 450. (2005). - Basónimo: Rondeletia hondurensis Donn. Sm., Bot. Gaz. 27: 335. (1899). - Holotipo: Honduras. Col.: Thieme (5267) (US) = Javorkaea hondurensis (Donn. Sm.) Borhidi et Járai-Komlódi, Acta Bot. Hung. 29: 16. (1983). - Ilustr.: Borhidi et Járai-Komlódi, Acta Bot. Hung. 29: 15. t. 1. (1983).

Arachnothryx izabalensis (Standl. et Steyerm.) Borhidi, Acta Bot. Hung. 33: 302. (1987). - Basónimo: Rondeletia izabalensis Standl. et Steyerm., Publ. Field Mus. Nat. Hist. Bot. Ser. 22: 286. (1940). - Holotipo: Guatemala. Col.: Standley (72891) (F). = Renistipula izabalensis (Standl. et Steyerm.) Borhidi, Acta Bot. Hung. 46: 123. (2004). - Según los estudios moleculares de Stranczinger et al. $(2010,2014)$ el género Renistipula pertenece a la tribu Hamelieae, a la cercania de Omiltemia Standl. mientras el género Arachnothryx pertenece a la tribu Guettardeae (Rova et al. 2009, Mans y Bremer 2010).

\section{LITERATURA CONSULTADA}

Andersson, L. y Rova, J. H. E. (1999): The rps16 intron and the phylogeny of the Rubiaceae. - Plant Syst. Evol. 214: 161-186. https://doi.org/10.1007/bf00985737

Bentham, G. y Hooker, W. J. (1873): Rubiaceae. - Genera Plantarum 2: 7-151.

Borhidi, A. (1982): Studies in Rondeletieae (Rubiaceae), III. The genera Rogiera and Arachnothryx. - Acta Bot. Acad. Sci. Hung. 28: 65-72.

Borhidi, A. (1987): Studies in Rondeletieae (Rubiaceae), X. New combinations of Central American taxa. - Acta Bot. Hung. 33: 301-303.

Borhidi, A. (1989): Studies in Rondeletieae (Rubiaceae), XI. Critical notes on some Central American species of Rondeletia s.1. - Acta Bot. Hung. 35: 309-312.

Borhidi, A. (1993): Problems and views in the taxonomy of the Rondeletia complex (Rubiaceae). - Intern. Conf. Syst. Rubiaceae, Oct. 4-6. 1993. Missouri Bot. Gard. Abstracts, pp. 9-10.

Borhidi, A. (1995): Studies in Rondeletieae (Rubiaceae), XII. New combinations of Mexican and Central American taxa. - Acta Bot. Hung. 38: 139-142. (1993-94).

Borhidi, A. (1999): Studies in Rondeletieae (Rubiaceae) XIII. New combinations of Mexican and Central American plants. - Acta Bot. Hung. 40: 15-16. (1996-97).

Borhidi, A. (2001): Additions and corrections to the "Nomenclator of Mexican and Central American Rubiaceae." - Acta Bot. Hung. 43: 37-78. https://doi.org/10.1556/ abot.43.2001.1-2.3

Borhidi, A. (2005): Estudios sobre Rubiáceas Mexicanas I. Dos especies y una variedad nuevas de los géneros Arachnothryx Planch y Rogiera Planch. en Chiapas. - Acta Bot. Hung. 47: 25-31. https://doi.org/10.1556/abot.47.2005.1-2.3

Borhidi, A. (2006): Rubiáceas de México. - Akadémiai Kiadó, Budapest, 512 pp. 
Borhidi, A. y Barreto Valdés, A. (2002): Introducción a la taxonomía de la familia Rubiaceae en la flora de México. - Acta Bot. Hung. 44: 237-280. https://doi.org/10.1556/ abot.44.2002.3-4.5

Borhidi, A. y Darók, J. (2001): A Rubiaceae család rendszertana. (The taxonomy of the family Rubiaceae). - In: Darók, J. (ed.): Taxonómiai és anatómiai tanulmányok a Rubiaceae családban. (Taxonomic and anatomical studies in the family Rubiaceae). Univ. Pécs, Bot. Dep. Publ, Pécs, pp. 187-214.

Borhidi, A. y Diego-Pérez, N. (2008): Flora de Guerrero. No. 35. Coussareae, Gardenieae, Hedyotideae, Mussendeae, Naucleae, Rondeletieae (Rubiaceae). - Fac de Ciencias, UNAM, 122 pp.

Borhidi, A. y Diego-Pérez, N. (2009): Estudios sobre Rubiaceas Mexicanas XXI. Una nueva especies de Arachnothryx Planch. (Rondeletieae) en Guerrero. - Acta Bot. Hung. 51: 11-14. https://doi.org/10.1556/abot.51.2009.1-2.2

Borhidi, A. y Fernández M. Z. (1981a): Studies in Rondeletieae (Rubiaceae) I. A new genus: Roigella. - Acta Bot. Acad. Sci. Hung. 27: 309-312.

Borhidi, A. y Fernández M. Z. (1981b): Studies in Rondeletieae (Rubiaceae) II. A new genus: Suberanthus. - Acta Bot. Acad. Sci. Hung. 27: 313-316.

Borhidi, A. y Járai-Komlódi, M. (1983): Studies in Rondeletieae (Rubiaceae), IV. A new genus: Javorkaea. - Acta Bot. Acad. Sci. Hung. 29: 13-27.

Borhidi, A. y Martínez-Salas, E. (2013): Estudios sobre Rubiáceas Mexicanas XLII. Una nueva especie del género Arachnothryx (Rubiaceae, Guettardeae) en Jalisco, México. - Acta Bot. Hung. 55: 223-226. https://doi.org/10.1556/abot.55.2013.3-4.3

Borhidi, A. y Velazco Gutierrez, K. (2012): Estudios sobre Rubiáceas Mexicanas XXXVI. Dos especies nuevas en el género Rogiera Planch. (Rondeletieae). - Acta Bot. Hung. 54: 51-58. https://doi.org/10.1556/abot.54.2012.1-2.5

Borhidi, A., Darók, J. y Stranczinger, Sz. (2011): Donnellyanthus (Rubiaceae, Rondeletieae) a new genus in the flora of Mexico and Meso-America. - Acta Bot. Hung. 53: 273-281. https://doi.org/10.1556/abot.53.2011.3-4.9

Borhidi, A., Martínez Salas, E. y Ramos Álvarez, C. H. (2015): Estudios sobre las Rubiáceas de México XLIX. Una nueva especie del género Arachnothryx (Rubiaceae, Guettardeae) en la flora de Veracruz. - Acta Bot. Hung. 57: 17-22. https://doi.org/10.1556/ abot.57.2015.1-2.4

Borhidi, A., Guadarrama, M. de los A., Ortiz, G. G. y Jiménez, N. del C. (2006): Estudios sobre Rubiáceas Mexicanas VII. Tres especies nuevas del Arachnothryx Planch. (Rondeletieae) de Oaxaca y Tabasco. - Acta Bot. Hung. 48: 39-41. https://doi.org/10.1556/ abot.48.2006.1-2.4

Borhidi, A., Darók, J., Kocsis, M., Stranczinger, Sz. y Kaposvári, F. (2004): El Rondeletia complejo en México. - Acta Bot. Hung. 46: 91-135. https://doi.org/10.1556/ABot.46.2004.1-2.8

Bremekamp, C. E. B. (1966): Remarks on the position, the delimitation and the subdivision of the Rubiaceae. - Acta Bot. Neerl. 15: 1-33. https://doi.org/10.1111/j.1438-8677.1966. tb00207.x

Bremer, B. (1992): Phylogeny of Rubiaceae based on molecular and morphological data. Ann. Mo. Bot. Gard. 79: 380-387. https://doi.org/10.2307/2399775

Bremer, B. (2009): A review of molecular phylogenetic studies of Rubiaceae. - Ann. Mo. Bot. Gard. 96: 4-26. https://doi.org/10.3417/2006197

Bremer, B., Andreasen, K. y Olsson, D. (1995): Subfamilial and tribal relationships in the Rubiaceae based on rbcL sequence data. - Ann. Mo. Bot. Gard. 82: 383-397. https://doi. org/10.2307/2399889 
Burger, W. C. y Taylor, C. M. (1993): Family \# 202 Rubiaceae. In: Burger, W. C. (ed.): Flora Costaricensis. - Fieldiana, Bot. n.s., 33: 1-333.

Croat, T. B. (1978): Rubiaceae. - In: Flora of Barro Colorado Island, pp. 791-828.

Curtis, W. (1851): Botanical Magazine 77. t. No. 7549.

De Candolle, A. P. (1830): Rubiaceae. - Prodr. Syst. Nat. Reg. Veget., Paris, 4: 341-622.

Darók, J. (2001a): Taxonómiai és anatómiai tanulmányok a Rubiaceae családban. (Taxonomic and anatomical studies in the family Rubiaceae). - Univ. Pécs, Bot. Dep. Publ., Pécs, 257 pp.

Darók, J. y Borhidi, A. (2000): Application of leaf epidermal morphology to taxonomic delimitations in the genus Javorkaea Borhidi \& Járai-Komlódi (Rubiaceae). - Acta Bot. Hung. 42: 83-89.

Darók, J. y Kocsis, M. (2001): Az epidermiszsejtek tulajdonságainak taxonómiai jelentôsége. (Taxonomic importance of the epidermal cell characters in Rubiaceae). - In: Darók, J. (ed.): Taxonómiai és anatómiai tanulmányok a Rubiaceae családban. (Taxonomic and anatomical studies in the family Rubiaceae). Univ. Pécs, Bot. Dep. Publ., Pécs, pp. 151-170.

Delprete, P. G. (1999): Rondeletieae (Rubiaceae), part 1. - Flora Neotrop. Monogr. 77: 1-226.

Delprete, P. G. (2004): Rubiaceae. - In: Smith, N. P., Heald, S. V., Henderson, A., Mori, S. A. y Stevenson, D. W. (eds): Flowering plant families of the American tropics. New York Botanical Garden Press, Bronx, New York, USA/Princeton University Press, Princeton, New Jersey, USA, pp. 328-333.

Dwyer, D. J. (1980a): Rubiaceae. In: Woodson, Jr., R. E., Scheryl, R. W. \& coll., Flora of Panama. Part 1. - Ann. Mo. Bot. Gard. 67: 1-256.

Dwyer, D. J. (1980b): Rubiaceae. In: Woodson, Jr., R. E., Scheryl, R. W. \& coll., Flora of Panama. Part IX. Family 179. Rubiaceae. Part II. - Ann. Mo. Bot. Gard. 67: 257-522.

Fernandez, M. Z. (1995): Estudio taxonómico del género Rondeletia L. s. 1. (Rubiaceae) Acta Bot. Hung. 38: 47-138. (1993-94).

Fernandez, M. Z. y Borhidi, A. (1985): Studies in Rondeletieae (Rubiaceae), VIII. Nuevos taxa del género Rondeletia en Cuba. - Acta Bot. Hung. 31: 147-172.

Fernandez, M. Z. y Herrera P. O. (1983): Studies in Rondeletieae (Rubiaceae) VI. Estudio taxonómico de Rondeletia odorata Jacq. - Acta Bot. Hung. 29: 35-41.

Govaerts, R. (2016): World checklist of Rubiaceae. - Royal Bot. Gard., Kew, http://apps.kew. org./wcsp.

Hemsley, W. B. (1879): The genus Rondeletia. Some corrections and emendations in the synonymy of some of the species of Rondeletia. - Gard. Chron. 12: 235.

Kiehn, M. (1995): Chromosome survey of the Rubiaceae. - Ann. Mo. Bot. Gard. 82: 398-408. https://doi.org/10.2307/2399890

Kirkbride, J. H. jr. (1968): A revision of the Panamian species of Rondeletia (Rubiaceae). Ann. Mo. Bot. Gard. 55: 372-391. https://doi.org/10.2307/2395131

Kocsis, M. y Borhidi, A. (2003a): Petiole anatomical studies of some Rondeletia L. species. Acta Bot. Hung. 45: 339-343. https://doi.org/10.1556/abot.45.2003.3-4.8

Kocsis, M. y Borhidi, A. (2003b): Petiole anatomy of some Rubiaceae genera. - Acta Bot. Hung. 45: 345-353. https://doi.org/10.1556/abot.45.2003.3-4.9

Kocsis, M., Darók, J. y Borhidi, A. (2004): Comparative leaf anatomy and morphology of some neotropical Rondeletia (Rubiaceae) species. - Plant Syst. Evol. 248: 205-218. https://doi.org/10.1007/s00606-002-0144-0

Lorence, D. H. (1991): New species and combinations in Mexican and Central American Rondeletia (Rubiaceae). - Novon 1: 135-157. https://doi.org/10.2307/3391371

Lorence, D. H. (1994): New species in Mexican and Mesoamerican Rubiaceae. - Novon 4: 119-136. https://doi.org/10.2307/3391581 
Lorence, D. H. (1999): A Nomenclator of Mexican and Central American Rubiaceae. - Mo. Bot. Garden Press 73: 1-177.

Lorence, D. H. (2012): 8. Arachnothryx Planch. - In: Davidse, G., Sousa, M. S., Knapp, S. y Chiang, F. (eds): Flora Mesoamericana, Vol 4. part 2. pp. 16-37.

Lorence, D. H. y Castillo-Campos, G. (1988): Tres nuevas especies y una nueva combinación en el género Rondeletia (Rubiaceae, Rondeletieae) de Veracruz y Oaxaca, México. - Biotica, 13: 147-157.

Lorence, D. H. y Taylor, C. M. (2012): Rubiaceae. - In: Davidse, G., Sousa, M. S, Knapp, S. y Chiang, F. (eds): Flora Mesoamericana, Vol 4. part 2. pp. 1-288.

Lundell, C. L. (1943): New vascular plants from Texas, Mexico and Central America. Amer. Midland Nat. 29: 469-492. https://doi.org/10.2307/2420805

Manns, U. y Bremer, B. (2010): Towards a better understanding of intertribal relationships and stable tribal delimitations within Cinchonoideae s.s. (Rubiaceae). - Mol. Phyl. Evol. 56: 21-39. https://doi.org/10.1016/j.ympev.2010.04.002

Martinez-Cabrera, D., Terrazas, T. y Ochoterena, H. (2009): Foliar and petiole anatomy of tribe Hamelieae and other Rubiaceae. - Ann. Mo. Bot. Gard. 96: 133-145. https://doi. org/10.3417/2006196

Motley, T. J, Wurdack, K. J. y Delprete, P. G. (2005): Molecular systematics of the Catesbeeae-Chiococceae complex (Rubiaceae) flower and fruit evolution and biogeographic implications. - Amer. J. Bot. 92: 316-329. https://doi.org/10.3732/ajb.92.2.316

Planchon, A. P. (1849): Flores des Serres. 5: 442-445.

Rova, J. H. E. (1999a): The Rondeletieae-Condamineeae-Sipaneeae complex (Rubiaceae). Diss. Bot. Inst., Göteborg Univ., pp. 7-29.

Rova, J. H. E. (1999b): Rubiaceae phylogeny based on rps16 sequence data. - Diss. Bot. Inst., Göteborg Univ. Suppl. II: 1-29.

Rova, J. H. E., Delprete, P. G., Andersson, L. y Albert, V. A. (1999): Rubiaceae phylogeny based on trnL-F sequence data. - Diss. Bot. Inst., Göteborg Univ., Suppl. I: 1-35.

Rova, J. H. E., Delprete, P. G., Andersson, L. y Albert, V. A. (2002): A trnL-F cpDNA sequence study of the Condamineae-Rondeletieae-Sipaneeae complex with implications on the phylogeny of Rubiaceae. - Amer. J. Bot. 89(1): 145-159. https://doi. org/10.3732/ajb.89.1.145

Rova, J. H. E., Delprete, P. G. y Bremer, B. (2009): The Rondeletia complex (Rubiaceae): An attempt to use ITS, rps16, and trnL-F sequence data to delimit Guettardeae, Rondeletieae and sections within Rondeletia. - Ann. Mo. Bot. Gard. 96: 182-193. https://doi. org/10.3417/2006179

Schumann, K. (1891): Rubiaceae. - In: Engler, A. y Prantl, K. (eds): Die Natürlichen Pflanzenfamilien. Nachtr. II-IV. Teil. Engelmann, Leipzig, pp. 309-316.

Standley, P. C. (1918-1934): Rubiaceae. - North American Flora 32: 1-300.

Standley, P. C. y Williams, L. O. (1975): Rubiaceae. In: Flora de Guatemala. - Fieldiana: Bot. 24(11): 1-274.

Steyermark, J. A. (1967): Rubiaceae. In: Maguire, B. y Wurdack, J. J. (eds): Botany of the Guayana Highland, part VII. - Mem. N. Y. Bot. Gard. 17: 178-285.

Steyermark, J. A. (1972): Rubiaceae. In: Maguire, B. y Wurdack, J. J. (eds): Botany of the Guayana Highlands. Part IX. - Mem. N. Y. Bot. Gard. 23: 227-832.

Steyermark, J. A. (1974): Rubiaceae. - In: Lasser, T. y Steyermark, J. A. (eds): Flora de Venezuela. Caracas, Inst. Bot. Vol. 9(1-3), pp. 1-2070. 
Stranczinger, Sz., Borhidi, A. y Szentpéteri, J. (2006): Phylogenetic relationships among some species of the Rondeletia-complex (Rubiaceae). - Acta Bot. Hung. 48: 427-434. https://doi.org/10.1556/abot.48.2006.3-4.15

Stranczinger, Sz., Galambos, A. y Borhidi, A. (2010): Phylogenetic study on genus and species levels on the Deppea complex (Hamelieae section). - Abstracts, Vth Intern. Rubiaceae and Gentianales Conf., Stockholm, 6-10 September, p. 58.

Stranczinger, Sz., Galambos, A., Szénásy, D. y Szalontai, B. (2014): Phylogenetic relationships in the Neotropical tribe Hamelieae (Rubiaceae, Cinchonoideae) and comments on its generic limits. - J. Syst Evol. 52: 643-650. https://doi.org/10.1111/jse.12103

Taylor, C. M. (2001): Rubiaceae Juss. In: Stewens, W. D. et al. (eds): Flora de Nicaragua. Ann. Mo. Bot. Gard. 85: 2206-2284.

Torres-Montúfar, A. y Ochoterena, H. (2013): Dos especies nuevas de la familia Rubiaceae de la sierra de los Tuxtlas, Veracruz, México. - Rev. Mex. Biodiv. 84: 1082-1089. https:// doi.org/10.7550/rmb.32503

Urban, I. (1900-1928): Symbolae Antillanae. Vols I-IX. - Leipzig.

Vales, M. A. (1983): Studies in Rondeletieae (Rubiaceae), VII. The significance of leaf epidermis for taxonomy in Neomazaea s. 1. - Acta Bot. Hung. 29: 43-53. 